\title{
Magmatic-hydrothermal evolution in a fractionating granite: a microchemical study of the Sn- W-F mineralized Mole Granite (Australia)
}

\section{Journal Article}

Author(s):

Audétat, Andreas; Günther, Detlef; Heinrich, Christoph A. (D)

Publication date:

2000-10

\section{Permanent link:}

https://doi.org/10.3929/ethz-b-000118845

\section{Rights / license:}

Creative Commons Attribution-NonCommercial-NoDerivatives 4.0 International

Originally published in:

Geochimica et Cosmochimica Acta 64(19), https://doi.org/10.1016/S0016-7037(00)00428-2 
This is the Green Access version of: Audétat, A., Günther, D. and Heinrich, C.A., 2000. Magmatichydrothermal evolution in a fractionating granite: A microchemical study of the Sn-W-Fmineralized Mole Granite (Australia). Geochimica et Cosmochimica Acta, vol. 64, pp. 3373-3393. https://doi.org/10.1016/S0016-7037(00)00428-2

\title{
Magmatic-hydrothermal evolution in a fractionating granite: A microchemical study of the Sn-W-F-mineralized Mole Granite (Australia)
}

\author{
A. Audétat ${ }^{1}$, D. Günther ${ }^{2}$ and C. A. Heinrich \\ Isotope Geology and Mineral Resources, Department of Earth Sciences, \\ ETH Zentrum NO, CH-8092 Zürich, Switzerland \\ ${ }^{1}$ present address for correspondence: Department of Geological Sciences, Virginia Tech, \\ Blacksburg VA 24061, U.S.A., geomail2@vt.edu \\ 2present address: Laboratory of Inorganic Chemistry, ETH Zentrum CAB, CH-8092 Zürich, \\ Switzerland
}

\begin{abstract}
A large granitic pluton associated with numerous hydrothermal ore deposits (the Mole Granite, northeastern New South Wales, Australia) was used for an integrated study of the chemical evolution of silicate melts and aqueous fluids during the late-magmatic to early-hydrothermal transition. Major and trace-element compositions were obtained by EPMA and Laser-Ablation ICPMS microanalysis of fluid inclusions and crystallized melt inclusions in magmatic phenocrysts and minerals from miarolitic cavities. Together with fluid-compositional data from the ore veins, these data allowed reconstruction of the evolving silicate melts and therefrom exsolving single-phase (supercritical) or two-phase (brine + vapor) fluids, from the time of initial fluid saturation through to the final solidification of F-rich residual melts. The analytical data and a Rayleigh fractionation model combining experimental partitioning data with constraints from the natural system demonstrate that the phase state, the salinity, and the ore-metal contents of the exsolving fluids vary dramatically with increasing degree of crystallization. Fluid properties vary even without variation of externally imposed parameters such as pressure or temperature, mainly because of the progressive enrichment of $\mathrm{F}$ in the melt. Early-saturating fluids are Cl-rich and immediately separate into coexisting brine and vapor phase due to the low pressure of the system $(\approx 1 \mathrm{kbar})$. Increasing $\mathrm{F}$ content of the melt reduces the partitioning of $\mathrm{Cl}$ to the fluid, such that later exsolving fluids are single-phase and of low salinity (at unchanged pressure conditions). Due to the contrasting complexation behavior of different ore metals, this evolution leads to a significant change in trace-metal partitioning as magma crystallization proceeds. The resulting variation in fluid compositions in turn controls the major variation in ore-metal ratios observed in the ore deposits (notably $\mathrm{Sn} / \mathrm{W}$ in this case). This conclusion agrees with recent data from porphyry-style systems, and indicates more generally that the magmatic-to-hydrothermal transition probably exerts the dominant control on the metal content of high-temperature hydrothermal ore deposits.
\end{abstract}




\section{Introduction}

Fluid saturation in hydrous silicate magmas is a critical process in the evolution of crustal igneous intrusions of intermediate to acid composition. It controls the depth and mode of pluton emplacement (Burnham, 1979; Burnham and Ohmoto, 1980; Shinohara and Hedenquist, 1997) and the occurrence and style of extrusive volcanism (Mader et al., 1994). Fluid saturation has profound consequences for the redistribution of volatiles and many trace elements in magmatichydrothermal systems (Candela and Holland, 1986; Candela, 1992; Lowenstern, 1994), and is generally considered to represent one of the essential steps in forming economic enrichments of $\mathrm{Cu}, \mathrm{Mo}, \mathrm{Sn}, \mathrm{W}, \mathrm{Au}$ and many other metals (Ishihara and Takenouchi, 1980; Stein and Hannah, 1990; Cline and Bodnar, 1991; Hedenquist and Lowenstern, 1994; Kesler, 1994; Shinohara, 1994; Candela and Piccoli, 1995; Thompson, 1995; Barnes, 1997; Ulrich et al., 1999).

While the influence of certain factors during the early magmatic stage (e.g., Sawkins, 1990; Blevin and Chappell, 1992) and the subsolidus hydrothermal stage (e.g., Heinrich, 1990) are understood relatively well, much less is known about the transition stage, i.e., the time between the beginning of fluid exsolution from a crystallizing melt and its final solidification. Our current knowledge of the process of fluid separation from crystallizing magmas is dominantly based on experimental studies of the partitioning of volatiles and trace metals among melts, fluids and minerals (London et al., 1989; Webster and Holloway, 1990; Keppler and Wyllie, 1991) and masstransfer models based on such experiments (Candela and Holland, 1986; Cline and Bodnar, 1991; Shinohara, 1994; Candela and Piccoli, 1995). Insights into the late-magmatic to earlyhydrothermal processes have also been gained by the study of fossil and active volcanic systems (Lowenstern, 1994; Vivo and Frezzotti, 1994; Webster and Duffield, 1994; Lowenstern, 1995; Rapien et al., 1997), and of plutonic equivalents at relatively shallow depth (Frezzotti, 1992; Yang and Bodnar, 1994), but there is a dearth of field-based quantitative observations on the behavior of trace-elements during the progressive separation of complex fluids from a fractionally crystallizing silicate melt.

The Mole Granite in eastern Australia is one of the most rigorously investigated 'natural laboratories' for hydrothermal processes associated with evolved granitic intrusions (Mulholland, 1943; Lonergan, 1971; Weber, 1974; Eadington, 1977, 1983; Stegman, 1983; Brodie, 1983; Cozens, 1984; Kleeman, 1982; Plimer and Kleeman, 1985; Sun and Eadington, 1987; Rankin et al., 1992; Heinrich et al., 1992; Heinrich and Ryan, 1992; Kleeman et al., 1997; Audétat et al., 1998; Audétat, 1999; Audétat et al., 1999; Henley et al., 1999, and numerous reports of the Geological Survey of New South Wales). Most of these studies deal with processes operating at subsolidus conditions, preserved in the large number of hydrothermal ore deposits associated with this intrusion (Fig. 1). Recent LA-ICP-MS microanalysis of fluid inclusions allowed a quantification of the contributions of cooling, boiling, wall-rock reaction and mixing between magmatic and meteoric fluids to the precipitation of ore minerals and to the large-scale zonation of specialized ore deposits around the pluton (Audétat et al., 1998, 1999). This paper reports the first major study linking the composition of hydrothermal fluids to the chemistry of a natural evolving magma, based on microanalysis of coexisting fluid and silicate melt inclusions in variably evolved rock portions. It is shown that interactions among variably incompatible volatile components $\left(\mathrm{H}_{2} \mathrm{O}, \mathrm{Cl}\right.$ and in this case especially F) control the phase state and the ore-metal content of the fluids, which in turn determines the ore-metal content of the hydrothermal ore deposits. 


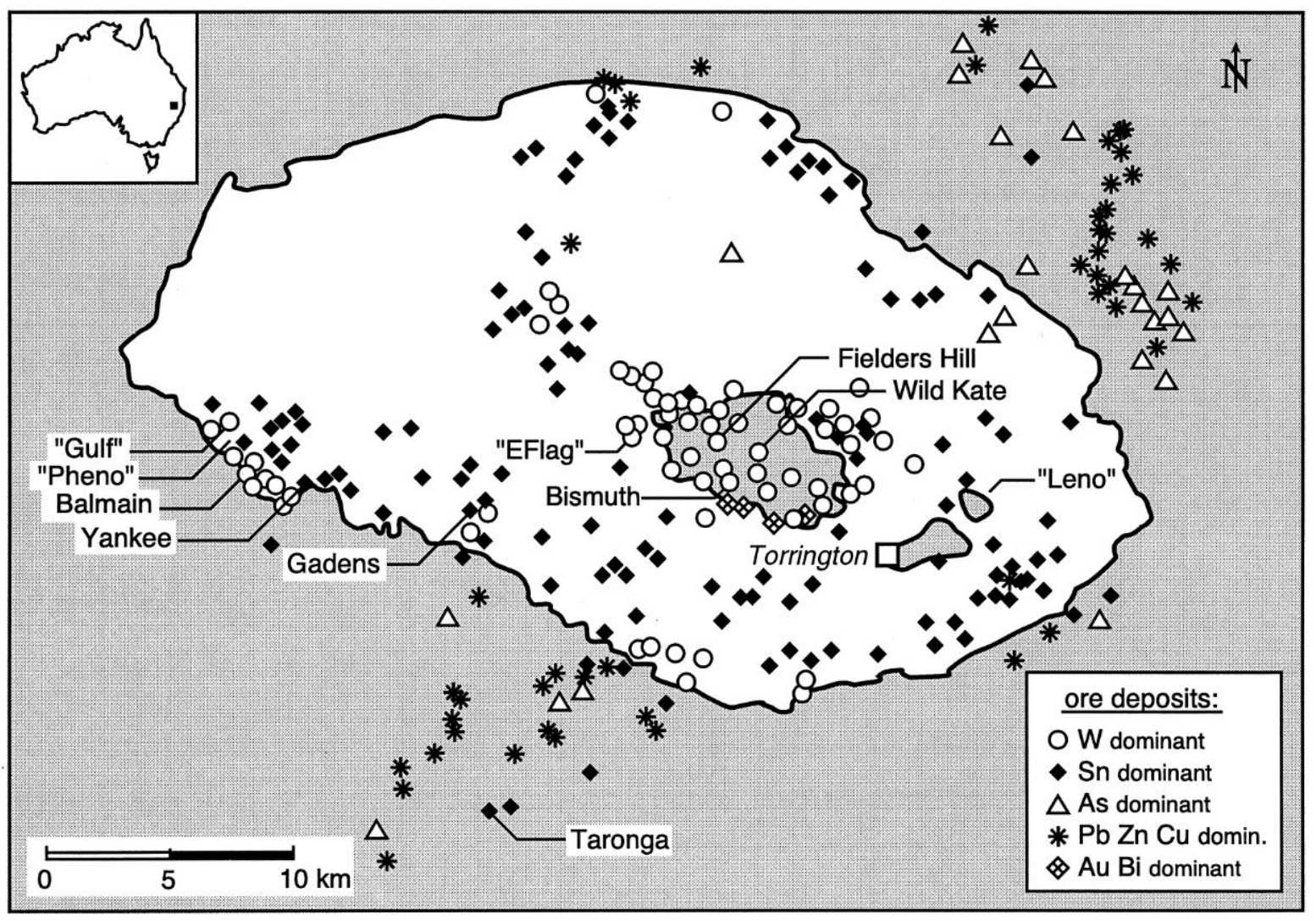

Figure 1. Distribution of hydrothermal ore deposits in and around the Mole Granite, based on a recent investigation by the New South Wales Geological Survey (Henley et al., 1999). Note that W-dominated deposits occur preferentially at the granite margin, whereas Sn-dominated typically occur in the more deeply eroded parts of the intrusion.

\section{Geological setting}

\subsection{Regional geology}

The Mole Granite is part of the large New England Batholith which extends over more than 400 $\mathrm{km}$ along the east coast of Australia. This batholith formed between $300 \mathrm{Ma}$ and $210 \mathrm{Ma}$ along an Andean-type continental margin and consists of a large number of individual plutons which intruded in several phases into a highly deformed subduction complex (Shaw and Flood, 1981; Godden, 1982; Fergusson and Leitch, 1993; Flood and Aitchison, 1993). Numerous authors attempted to subdivide the plutonic and volcanic rocks of this batholith into magmatic suites, based on geochemical and mineralogical criteria (Flinter et al., 1972; Shaw and Flood, 1981; Godden, 1982), isotopic signatures (Hensel et al., 1985) and radiometric ages (Shaw and Flood, 1993). The Mole Granite belongs to a series of leucogranites which are characterized by a high intrusion level, a minimum melt composition and high contents in $\mathrm{Li}, \mathrm{Rb}, \mathrm{U}$, Th and $\mathrm{F}$ (Weber, 1974; Juniper and Kleeman, 1979; Shaw and Flood, 1981; Hensel et al., 1985; Barnes et al., 1988; Vickery et al., 1997).

\subsection{Geology of the Mole Granite}


The strongly mineralized Mole Granite (Fig. 1) is only partially unroofed, with $\sim 650 \mathrm{~km}^{2}$ exposed surface and another $\sim 1200 \mathrm{~km}^{2}$ still buried under sedimentary and volcanic country rocks (Kleeman, 1982). Its age has been determined by K-Ar, $\mathrm{Ar}-\mathrm{Ar}$ and $\mathrm{Rb}-\mathrm{Sr}$ dating of whole rocks and hydrothermal vein minerals at $246 \pm 2$ Ma (Kleeman et al., 1997), confirmed by U-Pb ages of $246.5 \pm 1$ Ma recently measured on hydrothermal xenotime (pers. comm. Urs Schaltegger, ETH Zürich). Gravity modeling (Sardjono, 1988) and heat flow measurements (Kleeman, 1982) indicate that the Mole Granite forms a sill-like magma body of 1-4 km thickness. The exposed part of the intrusion is very homogeneous with respect to mineralogy and geochemistry (Table 1), suggesting that most of the magma intruded in a single event.

\subsubsection{Depth of emplacement}

Thickness estimates for the Emmaville Volcanics range from 2-4 km (Shaw and Flood, 1981; Godden, 1982; Shaw et al., 1988), whereas another $\sim 1 \mathrm{~km}$ is inferred for the overlying Dundee Rhyodacite (McPhie, 1988; Shaw et al., 1988). The Mole Granite should therefore have intruded at a depth of $4 \pm 1 \mathrm{~km}$, corresponding to a lithostatic pressure of $1.1 \pm 0.3 \mathrm{kbar}$ if an average density of $2.7 \mathrm{~g} / \mathrm{cm}^{3}$ is assumed. This value agrees well with a pressure of $0.9 \mathrm{kbar}$ established on coexisting brine- and vapor inclusions from the Yankee Lode, in which the pressure seems to have oscillated between lithostatic and near-hydrostatic conditions (Audétat et al., 1998). In an earlier fluid inclusion study, Eadington (1983) proposed a minimum pressure of 0.7 kbar. It should be noted, however, that pressure-estimates established on natural boiling have to be considered with caution, as they are calculated using the binary $\mathrm{H}_{2} \mathrm{O}-\mathrm{NaCl}$ model system.

\subsubsection{Igneous Petrology}

The Mole Granite is composed of three textural variants, which all are mineralogically and geochemically very similar. In decreasing order of abundance these are: seriate granite, porphyritic granite and microgranite (Table 1, Fig. 2a).

The porphyritic granite forms a 10-100 m thick layer in the apical portions of the intrusion. This layer seems to be thickest under flat-lying roof portions, whereas under the steeply dipping roof portions it is usually thin or even absent. The porphyritic granite contains phenocrysts of quartz (bipyramidal; max. $2 \mathrm{~cm}$ ), potassic feldspar (max. $4 \mathrm{~cm}$, some rimmed by albite), plagioclase (max. $2 \mathrm{~cm})$ and biotite in a fine-grained $(<1 \mathrm{~mm})$ groundmass of the same minerals. Primary topaz is a characteristic accessory mineral. Despite the fact that the total phenocryst content is highly variable (5-70 vol\%; average $30 \mathrm{vol} \%$ ), the relative abundance of the major minerals is always about the same ( $\mathrm{qtz} \approx \mathrm{kfsp}>$ plag $>>$ bio ). The phenocryst-content is usually lowest in the uppermost part of the porphyritic carapace, and tends to increase towards the underlying seriate granite. Circular masses of porphyritic granite were observed at the intersection of hydrothermal vein systems, which points to a pressure-quenched origin of this rock type, as suggested earlier by Kleeman (1982).

The seriate granite contains the same phenocryst assemblage as the porphyritic granite, but has a coarser grained matrix $(>1 \mathrm{~mm})$ and does not contain any primary topaz. The contact between porphyritic and seriate can be either sharp or gradual over a few meters. In the adit of the Balmains Lode a large block of already solidified porphyritic granite appears to have sunk into the underlying crystal-melt mixture of the seriate granite, producing flow alignment of the kfspphenocrysts.

The microgranite is clearly the youngest phase, occurring as dikes and irregular masses within both other textural variants. Microgranitic dikes follow the same fracture sets that control the hydrothermal quartz veins. Large, sill-like masses of microgranite commonly occur at the granite 
margin and at the contact between seriate granite and porphyritic granite (Stegman, 1983). The microgranite is characterized by an equigranular, hypidiomorphic intergrowth of quartz, potassic feldspar, plagioclase and biotite of usually less than $1 \mathrm{~mm}$ grain size. Magmatic topaz is abundant, and can make up several percent of the total rock volume (Stegman, 1983; Brodie, 1983; Cozens, 1984; Kleeman, 1982; and own observations).
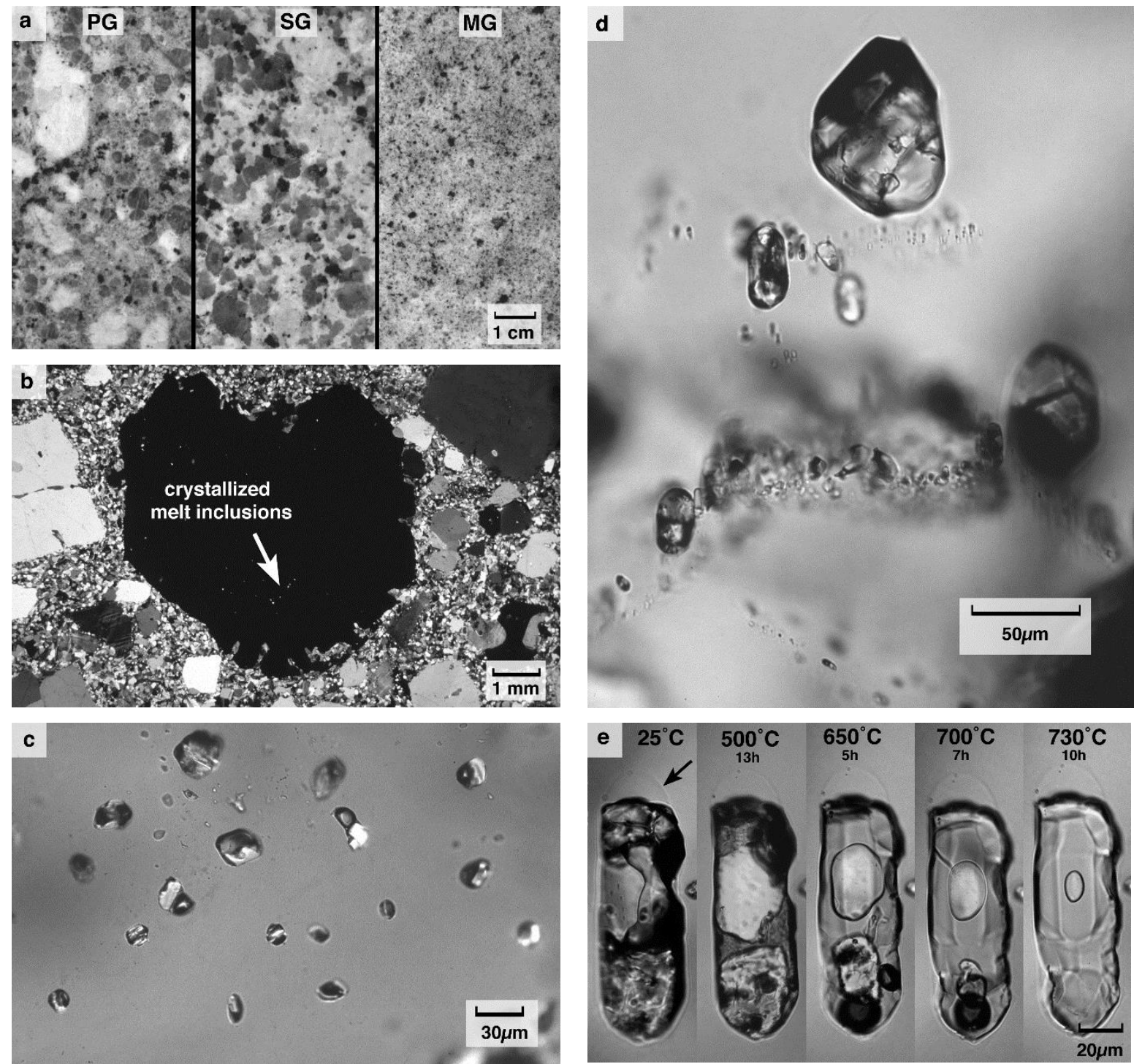

Figure 2. (a) Photograph of the three textural variants of the Mole Granite: (from left) phenocryst-rich porphyritic granite (PG), seriate granite (SG) and microgranite (MG). (b) Thin section of porphyritic granite, containing phenocrysts of quartz, potassic feldspar and plagioclase in a fine-grained matrix of the same minerals. The large quartz phenocryst in the center (black due to complete extinction) displays typical embayment structures, whereas crystallized melt inclusions appear as clouds of white dots. Transmitted light with crossed polarizers. (c) Close-up view of crystallized melt inclusions in a quartz phenocryst similar to that shown in Fig. $2 \mathrm{~b}$. Transmitted light with crossed polarizers. (d) Photo-micrograph of crystallized melt inclusions in magmatic topaz. Most of the inclusions were trapped along two former crystal surfaces that extend approximately horizontal in the picture (plain transmitted light). (e) Typical melting behavior of melt inclusions in topaz. The arrow points to a former outline of the inclusion, indicating that precipitated topaz did either not dissolve during the melting process, or dissolved at other places. The rounded mineral in the middle of the inclusion is quartz. Transmitted light with crossed polarizers.

Mafic microgranitoid enclaves occur within a narrow zone extending from the Butler Mine about $10 \mathrm{~km}$ to the west, generally close to the basal contact of the porphyritic granite to the 
underlying seriate granite. These enclaves contain the same phenocryst assemblage of quartz, potassic feldspar (some of them rimmed by albite), plagioclase and biotite as the surrounding granite, which is interpreted to result from a transfer of phenocrysts from the granitic melt crystal mixture into the mafic melt globules (Vernon, 1986; Didier and Babarin, 1991).

\subsubsection{Hydrothermal mineralization}

More than 1200 hydrothermal ore deposits are recorded in the Mole Granite district (Henley et al., 1999), most of which having been mined for either $\mathrm{Sn}$ or $\mathrm{W} / \mathrm{Bi}$, and a smaller number for $\mathrm{Cu}$, $\mathrm{Pb}, \mathrm{As}, \mathrm{Zn}, \mathrm{Ag}$ or $\mathrm{Au}$ (Fig. 1). Except for some large, mineralized fault systems, all ore deposits are confined to within 100-200 m vertical distance from the granite contact (Lonergan, 1971; Kleeman et al., 1997). More deeply eroded parts of the granite (e.g. the deep valleys $\mathrm{N}$ and NW of the sedimentary roof pendant) are not mineralized. Mineralization within the granite occurs mostly in quartz veins, or as disseminations in massive quartz \pm topaz greisens ("silexites"; Eadington and Nashar, 1978) at the granite margin. Ore deposits outside the granite include pegmatites, sheeted veins (above ridges or cupolas in the roof), and mineralized faults and shear zones.

There is a prominent metal zonation from $\mathrm{Sn}$-dominated deposits $( \pm \mathrm{B}, \mathrm{Cu}, \mathrm{Pb}, \mathrm{Zn})$ within the granite, through $\mathrm{W}$-dominated deposits $( \pm \mathrm{F}, \mathrm{Be}, \mathrm{Bi}, \mathrm{As}, \mathrm{Mo})$ at the granite margin, to sulfide-rich polymetallic deposits (As, $\mathrm{Pb}, \mathrm{Zn}, \mathrm{Cu}, \mathrm{Sb}, \mathrm{Ag}$ ) in the surrounding sediments (Fig. 1; see also Lonergan, 1971, and Henley et al., 1999). In a paper closely related to the herein presented work Audétat et al. (1999) discuss the reasons for this large-scale metal zonation. It is concluded that it reflects both a compositional variation of the source fluid, as well as sequential metal precipitation from it.

The Mole Granite hosts some of the world's largest quartz-topaz greisens, characterized by average contents of 20-30 vol\% topaz, 0.1-0.3 wt $\% \mathrm{WO}_{3}$, and $0.05 \mathrm{wt} \% \mathrm{Bi}$ (McClatchie, 1981; Creech, 1992). Eadington and Nashar (1978) proposed a magmatic origin of these rocks, based on analyses of supposed melt inclusions within topaz. However, the "glassy melt inclusions" described by these workers are remains of minerals that were replaced during the greisenization, marking the border between primary magmatic topaz and metasomatic overgrowths. True melt inclusions occurring in the magmatic cores are totally crystallized, and are identical (in terms of composition and melting behavior) to topaz-hosted melt inclusions in unaltered microgranite. Further evidence for a dominantly metasomatic origin of the quartz-topaz rocks is given by the field relationships discussed by Kleeman (1985), Kleeman et al. (1997) and Audétat et al. (1999). Descriptions of individual ore deposits associated with the Mole Granite can be found in Mulholland (1943), Lonergan (1971), Weber (1974), Eadington (1977), Stegman (1983), Brodie (1983), Cozens (1984) and Audétat et al. (1999), whereas summaries are given by Eadington (1983), Plimer and Kleeman (1985), Kleeman et al. (1997) and Henley et al. (1999).

\section{Methods}

The subsolidus hydrothermal stage in the evolution of the Mole Granite has been investigated with a number of fluid-inclusion studies on selected vein and greisen deposits. Quartz crystals containing several generations of fluid inclusions were examined in great detail, allowing differentiation between inclusions that were trapped before, during and after the precipitation of ore minerals. By analyzing selected inclusions by LA-ICP-MS we could reconstruct the compositional evolution of the fluid for the case of a Sn-deposit (Yankee Lode), a W-deposit (EFlag), and a miarolitic cavity (Leno 1)(Audétat et al., 1998; 1999). In the present paper we complement these investigations on the subsolidus stage with data from silicate melt inclusions and selected fluid inclusions that coexisted with the evolving granite melt. Using petrographic observations and 
LA-ICP-MS analysis of coexisting melt and fluid inclusions we aim at determining (i) at which degree of crystallization the fluid exsolution took place, (ii) which was the physical state of the fluid (one-phase vs. two-phase), and (iii) how the chemical composition of the fluid(s) and coexisting melt changed with increasing degree of crystallization. A Rayleigh fractionation model of melt - fluid evolution based on published experimental partitioning data is then developed to test and explain the combined microchemical data.

\subsection{Melting experiments}

Most petrographic observations were made on doubly polished sections of 200-500 $\mu$ m thickness. Quartz phenocrysts and magmatic topaz grains were often found to contain several generations of crystallized melt inclusions, trapped at various degrees of crystallization and chemical fractionation of the granite (Figs. 2b, c, d; Fig. 6). To help identification of different generations of quartz in the quartz phenocrysts, cathodoluminescence images (Marshall, 1988) were prepared using the technique described by Gebauer (1996). Fluid inclusion microthermometry was performed on a Linkam Stage THMSG 600 calibrated to an uncertainty of $\pm 0.05^{\circ} \mathrm{C}$ at $0{ }^{\circ} \mathrm{C}$, and $\pm 2{ }^{\circ} \mathrm{C}$ at $374.1^{\circ} \mathrm{C}$. Care was taken that only inclusions showing no signs of postentrapment modifications (Audétat and Günther, 1999) were selected.

Re-melting experiments of crystallized melt inclusions were carried out at atmospheric pressure in a tube furnace, and at 700 bar confining pressure in cold-seal bombs. The samples were kept at a given temperature for 2-70 hrs. Subsequent quenching occurred within 1-2 sec in the 1 bar experiments, and within $\sim 5 \mathrm{~min}$ in the high-pressure experiments. It was found that it takes about 8-10 hrs to attain crystal-melt equilibrium in an inclusion of 50-200 $\mu \mathrm{m}$ size, which is much faster than times reported in Thomas et al. (1996). This discrepancy may be explained by the higher fluorine content (and therefore lower viscosity) in the samples from the Mole Granite. The application of 700 bar confining pressure had no effect on the melting behavior, except that fewer inclusions were lost by decrepitation.

Melt inclusions hosted by quartz phenocrysts could not be re-homogenized, or if so only at very high temperatures $\left(>1000^{\circ} \mathrm{C}\right)$, indicating that they lost water and possibly also other components (see section 4.3). Melt inclusions hosted by topaz on the other hand showed very consistent and geologically reasonable homogenization temperatures between $700{ }^{\circ} \mathrm{C}$ and $740{ }^{\circ} \mathrm{C}$ (Figs. 2e, 3). Theyr low first melting temperatures $\left(<500{ }^{\circ} \mathrm{C}\right)$ are petrologically "meaningless", as they are (at least partially) caused by high internal pressures generated during the re-melting process (Student and Bodnar, 1996).

\section{2. $\quad$ LA-ICP-MS analysis of fluid- and melt inclusions}

Laser Ablation-ICP-MS was used to determine the chemical composition of more than 500 single fluid inclusions and 105 melt inclusions. This microanalytical technique has made enormous progress over the last few years, and now enjoys great popularity in various geological applications (Günther et al., 1997; Taylor et al., 1997; Günther et al., 1998). The major advantage in the analysis of crystallized melt inclusions lies in the fact that they do not have to be re-melted, nor to be exposed to the surface prior to analysis. Entire inclusions can be drilled out of the host mineral and be reconstituted by integration of the transient LA-ICP-MS signal. This not only saves preparation time, but also eliminates the common difficulties of achieving complete remelting of all solids during heating. Another advantage of LA-ICP-MS is the fast data acquisition, allowing simultaneous measurement of up to 40 major-, minor- and trace-elements in each inclusion within minutes. 
Table 1. Analysis of melt inclusions (LA-ICPMS/EPMA), fluid inclusions (LA-ICPMS) and whole-rocks (XRF/CPMS)

\begin{tabular}{|c|c|c|c|c|c|c|c|c|c|}
\hline Sample name & Description & Host & $\mathrm{NaCl}_{\text {equiv }}{ }^{\mathrm{a}}$ & $\mathrm{Th}_{\mathrm{tot}}$ & $\mathrm{n}^{\mathrm{b}}$ & $\mathrm{Li}$ & $\mathrm{B}$ & $\mathrm{F}$ wt.\% & Na wt.\% \\
\hline \multicolumn{10}{|l|}{ Whole rocks } \\
\hline Carp W1 & Seriate granite & & & & 1 & 40 & 11 & 0.50 & 2.43 \\
\hline Vnlt fresh & Porphyritic granite & & & & 1 & 44 & 12 & 0.35 & 2.43 \\
\hline Fiel3A & Tpz-bearing microgranite & & & & 1 & 104 & 17 & 0.56 & 2.64 \\
\hline $\begin{array}{l}\text { Mole granite } \\
\text { Melt inclusions }\end{array}$ & Average of 10 analyses & & & & 10 & $59 \pm 45$ & $10 \pm 2$ & $0.30 \pm 0.15$ & $2.44 \pm 0.12$ \\
\hline Pheno 3 & 1. growth zone & Quartz phenocryst in porph. granite & & & 7 & $314 \pm 40$ & $25 \pm 11$ & - & $0.11 \pm 0.01$ \\
\hline Pheno 3 & 2. growth zone & Quartz phenocryst in porph. granite & & & 6 & $214 \pm 62$ & $57 \pm 7$ & - & $0.14 \pm 0.03$ \\
\hline Pheno 3 & Embayment & Quartz phenocryst in porph. granite & & & 3 & $67 \pm 26$ & $2,916 \pm 861$ & - & $0.39 \pm 0.03$ \\
\hline Pheno 4 & Late growth zone (?) & Quartz phenocryst in porph. granite & & & 3 & $217 \pm 28$ & - & - & $0.11 \pm 0.01$ \\
\hline Pheno 4 & Embayment & Quartz phenocryst in porph. granite & & & 7 & & $461 \pm 51$ & - & $0.20 \pm 0.05$ \\
\hline Yank 8.1 & Dispersed inclusions & Matrix quartz in seriate granite & & & 3 & $47 \pm 9$ & - & - & $0.14 \pm 0.01$ \\
\hline Fiel 3 & Primary; no time rel. & Magm. topaz in microgranite & & & 3 & $292 \pm 154$ & $550 \pm 209$ & $2.94 * \pm 0.01$ & $0.60 \pm 0.73$ \\
\hline Fiel 10 & Primary; no time rel. & Magm. topaz in pegmatitic vein & & & 7 & $108 \pm 27$ & $623 \pm 154$ & $2.04 * \pm 0.04$ & $2.04 \pm 0.04$ \\
\hline Fiel 15 & Primary; no time rel. & Magm. topaz in pegmatitic vein & & & 14 & $115 \pm 45$ & $595 \pm 104$ & $1.89 * \pm 0.10$ & $1.89 \pm 0.10$ \\
\hline Fiel 30 & Primary; no time rel. & Qz-topaz vein (orig. pegmatitic?) & & & 8 & $171 \pm 42$ & $1,121 \pm 185$ & $2.26 * \pm 0.04$ & $1.33 \pm 0.94$ \\
\hline Bism 3.4 & 1. generation & Magmatic topaz in qtz-tpz greisen & & & 9 & $251 \pm 76$ & $668 \pm 60$ & - & $1.72 \pm 1.55$ \\
\hline Bism 3.4 & 2. generation & Magmatic topaz in qtz-tpz greisen & & & 1 & 580 & 627 & - & 0.10 \\
\hline Bism 3.4 & 3. generation & Magmatic topaz in qtz-tpz greisen & & & 2 & $275 \pm 137$ & $583 \pm 82$ & - & $0.09 \pm 0.03$ \\
\hline Bism 3.3A (a) & Coexisting with fluid (b) & Magmatic topaz in qtz-tpz greisen & & & 2 & $60 \pm 24$ & $400 \pm 61$ & - & $0.08 \pm 0.01$ \\
\hline \multicolumn{10}{|l|}{ Fluid inclusions } \\
\hline Bism 3.3A (b) & S'crit. fluid coexist. with (a) & Magmatic topaz in qtz-tpz greisen & 4.5 & - & 4 & $838 \pm 110$ & 1,841 & - & 1.38 \\
\hline Bism 3.4B & Early brine inclusions & Magmatic topaz in qtz-tpz greisen & $37.1 \pm 0.4$ & 540 & 3 & $2,056 \pm 215$ & $1,057 \pm 45$ & - & $14.60 \pm 015$ \\
\hline Bism 3.3A & Early brine inclusion & Magmatic topaz in qtz-tpz greisen & 40.7 & 563 & 1 & 595 & 971 & - & 9.85 \\
\hline EFlag2 & Pre-mineraliz. brine & Quartz crystal from W-bein & 39.0 & 350 & 1 & - & 1,160 & - & 8.93 \\
\hline Yank 20C & Pre-mineraliz. brine & Quartz crytal from Sn-vein & $2.81 \pm 0.3$ & 528 & 7 & $1,452 \pm 104$ & $2,437 \pm 208$ & - & $7.27 \pm 0.54$ \\
\hline Yank 20C & Coexisting vapor & Quartz crystal from Sn-vein & $6.3 \pm 0.8$ & - & 3 & $597 \pm 161$ & $3,472 \pm 641$ & - & $1.72 \pm 0.01$ \\
\hline Low w9 & Syn-mineraliz. brine & Quartz crystal from W-vein & $38.2 \pm 4.5$ & 437 & 9 & $1,057 \pm 378$ & $2,842 \pm 1,172$ & - & $5.08 \pm 2.44$ \\
\hline Low w9 & Syn-mineraliz. vapor & Quartz crystal from W-vein & $2.1 \pm 0.2$ & - & 8 & $454 \pm 177$ & $3,306 \pm 1,594$ & - & $0.65 \pm 0.11$ \\
\hline Wolf 12 & Syn-mineraliz. brine & Quartz crystal from W-greisen & $32.7 \pm 0.4$ & 400 & 2 & $433 \pm 97$ & $151 \pm 40$ & - & $7.23 \pm 1.08$ \\
\hline Wolf 12 & Coexisting vapor & Quartz crystal from W-greisen & 3.5 & - & 4 & 1,829 & - & - & $1.02 \pm 0.11$ \\
\hline
\end{tabular}


Table 1. (continued)

\begin{tabular}{|c|c|c|c|c|c|c|c|c|c|}
\hline Sample name & $\mathrm{Mg}$ & $\mathrm{Al}$ wt. $\%$ & Si wt.\% & $\mathrm{P}$ & $\mathrm{Cl}$ & K wt.\% & Ca wt. $\%$ & $\mathrm{Ti}$ & Mn \\
\hline \multicolumn{10}{|l|}{ Whole rocks } \\
\hline Carp W1 & 482 & 6.44 & 35.91 & 87 & 10 & 4.22 & 0.35 & 539 & 310 \\
\hline Vnlt fresh & 482 & 6.29 & 36.14 & 44 & 13 & 4.00 & 0.31 & 539 & 155 \\
\hline Fiel3A & 302 & 7.45 & 35.18 & 131 & 11 & 4.17 & 0.11 & 180 & 77 \\
\hline \multicolumn{9}{|l|}{ Melt inclusions } & $170 \pm 71$ \\
\hline Pheno 3 & $489 \pm 129$ & 6.35 & - & 59 & - & $2.96 \pm 0.15$ & $0.25 \pm 0.06$ & $956 \pm 126$ & $324 \pm 74$ \\
\hline Pheno 3 & $373 \pm 89$ & 6.35 & - & - & - & $2.67 \pm 0.17$ & - & $583 \pm 58$ & $330 \pm 80$ \\
\hline Pheno 3 & $36 \pm 3$ & 6.35 & - & - & - & $2.78 \pm 0.03$ & - & 265 & $365 \pm 132$ \\
\hline Pheno 4 & $391 \pm 295$ & 6.35 & - & - & - & $3.44 \pm 0.17$ & - & $783 \pm 101$ & $439 \pm 46$ \\
\hline Pheno 4 & $37 \pm 22$ & 6.35 & - & - & - & $2.83 \pm 0.34$ & - & - & $221 \pm 36$ \\
\hline Yank 8.1 & $174 \pm 49$ & 6.35 & - & - & - & $2.89 \pm 0.10$ & - & $416 \pm 31$ & $340 \pm 43$ \\
\hline Fiel 3 & $56 \pm 37$ & $7.49 * \pm 0.17$ & $31.33 * \pm 0.30$ & $180^{*}$ & $2335^{*} \pm 106$ & 3.20 & - & $197 \pm 121$ & $562 \pm 200$ \\
\hline Fiel 10 & $102 \pm 16$ & $8.12 \pm 0.10$ & - & $110 \pm 99$ & $2,660 \pm 71$ & 2.80 & - & $136 \pm 28$ & $505 \pm 36$ \\
\hline Fiel 15 & $84 \pm 11$ & $7.95 * \pm 0.26$ & $32.41 * \pm 0.61$ & $200^{*} \pm 99$ & $2728^{*} \pm 239$ & 3.15 & - & $90 \pm 24$ & $410 \pm 72$ \\
\hline Fiel 30 & $134 \pm 44$ & $7.93 * \pm 0.18$ & $31.94 * \pm 0.95$ & $220^{*}$ & $2884 * \pm 92$ & 3.50 & 0.31 & $316 \pm 89$ & $1,012 \pm 65$ \\
\hline Bism 3.4 & $17 \pm 5$ & - & - & - & - & 3.50 & - & $69 \pm 17$ & $962 \pm 216$ \\
\hline Bism 3.4 & 82 & - & - & 159 & - & 3.50 & 0.15 & 270 & 715 \\
\hline Bism 3.4 & 108 & - & - & - & - & 3.50 & - & $143 \pm 69$ & $732 \pm 195$ \\
\hline Bism 3.3A (a) & $57 \pm 34$ & - & - & - & - & - & $<0.2$ & $170 \pm 16$ & $367 \pm 5$ \\
\hline Fluid inclusions & & & & wt.\% & & & & wt.\% & \\
\hline Bism 3.3A (b) & - & - & - & - & - & $0.57 \pm 0.17$ & $<15.5$ & $<1395$ & $0.12 \pm 0.02$ \\
\hline Bism 3.4B & $48 \pm 32$ & - & - & $1,138 \pm 354$ & $27.99 \pm 0.59$ & $12.00 \pm 0.52$ & - & $568 \pm 383$ & $3.89 \pm 0.28$ \\
\hline Bism 3.3A & 73 & - & - & - & 39.17 & 13.56 & - & - & 3.97 \\
\hline EFlag2 & - & - & - & - & 23.50 & 5.42 & - & - & 1.48 \\
\hline Yank 20C & - & - & - & - & - & $3.54 \pm 0.42$ & - & - & $1.42 \pm 0.21$ \\
\hline Yank 20C & - & - & - & - & - & $0.61 \pm 0.10$ & - & - & $0.15 \pm$ \\
\hline Low w9 & - & $0.27 \pm 0.24$ & - & - & $58.72 \pm 17.18$ & $9.76 \pm 1.41$ & $0.49 \pm 0.21$ & - & $2.45 \pm 0.30$ \\
\hline Low w9 & - & $0.75 \pm 0.99$ & - & - & & $0.12 \pm 0.02$ & $0.62 \pm 0.41$ & - & $0.04 \pm 0.03$ \\
\hline Wolf 12 & $53 \pm 37$ & $0.02 \pm 0.00$ & - & - & $20.85 \pm 6.14$ & $4.56 \pm 0.07$ & - & - & $1.94 \pm 0.14$ \\
\hline Wolf 12 & $371 \pm 166$ & - & - & - & - & $0.41 \pm 0.12$ & - & & $0.13 \pm 0.03$ \\
\hline
\end{tabular}


Table 1. (continued)

\begin{tabular}{|c|c|c|c|c|c|c|c|c|c|}
\hline Sample name & Fe wt. $\%$ & $\mathrm{Cu}$ & $\mathrm{Zn}$ & As & $\mathrm{Rb}$ & $\mathrm{Sr}$ & $\mathrm{Y}$ & $\mathrm{Zr}$ & $\mathrm{Ag}$ \\
\hline \multicolumn{10}{|l|}{ Whole rocks } \\
\hline Carp W1 & 0.83 & 3 & 104 & 4 & 623 & 9 & 80 & 120 & - \\
\hline Vnlt fresh & 0.86 & 1 & 63 & 3 & 554 & 7 & 95 & 141 & - \\
\hline Fiel3A & 0.47 & 6 & 10 & 5 & 834 & 21 & 97 & 65 & - \\
\hline $\begin{array}{l}\text { Mole Granite } \\
\text { Melt inclusions }\end{array}$ & $0.71 \pm 0.36$ & $11 \pm 16$ & $39 \pm 27$ & $14 \pm 19$ & $584 \pm 127$ & $11 \pm 5$ & $87 \pm 27$ & $123 \pm 18$ & - \\
\hline Pheno 3 & $1.06 \pm 0.19$ & - & $52 \pm 19$ & $27 \pm 7$ & $579 \pm 106$ & $4.8 \pm 1.6$ & $43 \pm 14$ & $105 \pm 23$ & $0.4 \pm 0.4$ \\
\hline Pheno 3 & $0.72 \pm 0.10$ & - & $39 \pm 11$ & $26 \pm 5$ & $600 \pm 138$ & $5.5 \pm 1.9$ & $28 \pm 15$ & $93 \pm 38$ & - \\
\hline Pheno 3 & $0.19 \pm 0.05$ & - & $77 \pm 1$ & $383 \pm 448$ & $1,108 \pm 122$ & $2.6 \pm 1.3$ & $5 \pm 2$ & $11 \pm 11$ & - \\
\hline Pheno 4 & $0.82 \pm 0.08$ & - & 49 & $53 \pm 8$ & $937 \pm 152$ & $2.9 \pm 1.1$ & - & $107 \pm 27$ & $0.9 \pm 0.1$ \\
\hline Pheno 4 & - & - & - & $593 \pm 316$ & $876 \pm 216$ & $9.9 \pm 7.7$ & - & $9 \pm 4$ & 0.2 \\
\hline Yank 8.1 & $0.28 \pm 0.06$ & $13 \pm 1$ & $32 \pm 11$ & $96 \pm 25$ & $1,163 \pm 187$ & $3.4 \pm 0.0$ & - & $79 \pm 4$ & $1.6 \pm 0.6$ \\
\hline Fiel 3 & $0.60 \pm 0.38$ & $8 \pm 7$ & $29 \pm 2$ & $389 \pm 224$ & $2,664 \pm 963$ & $3.7 \pm 2.1$ & - & $75 \pm 86$ & $0.8 \pm 0.7$ \\
\hline Fiel 10 & $0.35 \pm 0.00$ & $3 \pm 0$ & $25 \pm 2$ & $358 \pm 25$ & $1,804 \pm 125$ & $6.3 \pm 2.8$ & - & $31 \pm 4$ & $0.3 \pm 0.2$ \\
\hline Fiel 15 & $0.19 \pm 0.03$ & $5 \pm 3$ & $17 \pm 1$ & $302 \pm 53$ & $1,216 \pm 180$ & $3.2 \pm 0.7$ & - & $9 \pm 3$ & $0.2 \pm 0.1$ \\
\hline Fiel 30 & $0.53 \pm 0.03$ & 6 & $25 \pm 3$ & $767 \pm 121$ & $1,913 \pm 128$ & $8.1 \pm 1.0$ & $191 \pm 39$ & $30 \pm 5$ & $0.4 \pm$ \\
\hline Bism 3.4 & $0.36 \pm 0.08$ & 8 & $24 \pm 5$ & $369 \pm 57$ & $3,064 \pm 149$ & $0.7 \pm 0.2$ & $38 \pm 9$ & $17 \pm 5$ & - \\
\hline Bism 3.4 & 0.55 & 3 & 28 & 275 & 3,296 & 0.9 & 15 & 28 & - \\
\hline Bism 3.4 & $0.62 \pm 0.25$ & 18 & $34 \pm 9$ & $399 \pm 92$ & $3,348 \pm 271$ & $2.6 \pm 0.2$ & $46 \pm 18$ & $20 \pm 3$ & - \\
\hline Bism 3.3A (a) & $0.21 \pm 0.00$ & $<22$ & $<137$ & $251 \pm 10$ & $2,992 \pm 306$ & 1.7 & $18 \pm 10$ & $15 \pm 0.4$ & $<1.9$ \\
\hline \multicolumn{10}{|l|}{ Fluid inclusions } \\
\hline Bism 3.3A (b) & 2.25 & $<336$ & $<2,500$ & $963 \pm 208$ & $455 \pm 79$ & $<25$ & $<15$ & $18 \pm 8$ & $<53$ \\
\hline Bism 3.4B & $17.94 \pm 1.24$ & $2,144 \pm 276$ & $9,365 \pm 1,006$ & $873 \pm 320$ & $9,719 \pm 198$ & $17 \pm 1$ & $6 \pm 3$ & 4 & $75 \pm 5$ \\
\hline Bism 3.3A & 21.58 & 1,120 & 7,312 & 1,017 & 8,185 & 59 & 6 & - & 85 \\
\hline EFlag2 & 8.23 & - & 5,388 & 400 & 2,142 & 44 & - & - & 24 \\
\hline Yank 20C & $4.02 \pm 1.01$ & $599 \pm 198$ & $3,186 \pm 787$ & $184 \pm 32$ & $1,404 \pm 270$ & - & - & - & $256 \pm 62$ \\
\hline Yank 20C & 0.44 & $2,304 \pm 423$ & $373 \pm 45$ & $190 \pm 30$ & $182 \pm 26$ & - & - & - & 108 \\
\hline Low w9 & $12.14 \pm 2.16$ & $231 \pm 162$ & $14,574 \pm 3,154$ & $221 \pm 80$ & $2,296 \pm 695$ & $31 \pm 12$ & - & - & $55 \pm 12$ \\
\hline Low w9 & $0.22 \pm 0.06$ & $3,065 \pm 1,269$ & $235 \pm 111$ & $214 \pm 78$ & $36 \pm 20$ & $2 \pm 2$ & - & - & $3 \pm 1$ \\
\hline Wolf 12 & $6.77 \pm 1.88$ & $165 \pm 10$ & $5,243 \pm 1,097$ & $167 \pm 60$ & $1,118 \pm 469$ & $174 \pm 69$ & - & - & $22 \pm 2$ \\
\hline Wolf 12 & $0.53 \pm 0.13$ & $2,594 \pm 1,474$ & $567 \pm 154$ & $71 \pm 35$ & $95 \pm 21$ & $14 \pm 2$ & - & - & - \\
\hline
\end{tabular}


Table 1. (continued)

\begin{tabular}{|c|c|c|c|c|c|c|c|c|c|c|c|c|}
\hline Sample name & Mo & Sn & $\mathrm{Sb}$ & Cs & $\mathrm{Ba}$ & $\mathrm{La}$ & $\mathrm{Ce}$ & W & $\mathrm{Pb}$ & $\mathrm{Bi}$ & Th & $\mathrm{U}$ \\
\hline \multicolumn{13}{|l|}{ Whole rocks } \\
\hline Carp W1 & 2 & 11 & 0.5 & 29 & 25 & 56 & 121 & 1.6 & 68 & 0.3 & 51 & 17 \\
\hline Vnlt fresh & 2 & 9 & 0.2 & 16 & 10 & 46 & 103 & 1.6 & 53 & 0.9 & 60 & 27 \\
\hline Fiel3A & 0.3 & 5 & 0.1 & 31 & 51 & 37 & 125 & 2.3 & 14 & 13 & 29 & 12 \\
\hline $\begin{array}{l}\text { Mole granite } \\
\text { Melt inclusions }\end{array}$ & $2 \pm 1$ & $6 \pm 3$ & $0.4 \pm 0.2$ & $21 \pm 10$ & $29 \pm 21$ & $44 \pm 10$ & $103 \pm 20$ & $1.4 \pm 0.4$ & $49 \pm 14$ & $1.2 \pm 1.2$ & $52 \pm 7$ & $20 \pm 11$ \\
\hline Pheno 3 & 2 & 57 & $3 \pm 2$ & $14 \pm 5$ & $47 \pm 4$ & $52 \pm 7$ & $115 \pm 16$ & $6 \pm 1$ & $14 \pm 8$ & $0.6 \pm 0.2$ & $41 \pm 5$ & $11 \pm 1$ \\
\hline Pheno 3 & - & - & $2 \pm 1$ & $35 \pm 8$ & $41 \pm 14$ & $55 \pm 6$ & $94 \pm 14$ & $6 \pm 1$ & $14 \pm 2$ & $0.6 \pm 0.1$ & $40 \pm 8$ & $11 \pm 1$ \\
\hline Pheno 3 & - & - & $35 \pm 6$ & $409 \pm 124$ & - & $26 \pm 10$ & $4 \pm 2$ & $10 \pm 3$ & $18 \pm 11$ & 5.5 & $6 \pm 1$ & $3 \pm 0.4$ \\
\hline Pheno 4 & - & - & $5 \pm 0.4$ & $107 \pm 127$ & - & $135 \pm 71$ & $113 \pm 83$ & $10 \pm 3$ & $22 \pm 7$ & $0.9 \pm 0.2$ & $34 \pm 25$ & $14 \pm 2$ \\
\hline Pheno 4 & - & $17 \pm 2$ & $18 \pm 11$ & $158 \pm 85$ & - & $4 \pm 3$ & $9 \pm 6$ & $34 \pm 11$ & $24 \pm 9$ & $1.4 \pm 0.9$ & $5 \pm 2$ & $6 \pm 1$ \\
\hline Yank 8.1 & - & - & $9 \pm 2$ & $253 \pm 33$ & - & $52 \pm 0.4$ & $136 \pm 25$ & $19 \pm 2$ & $29 \pm 2$ & $1.4 \pm 0.2$ & $46 \pm 4$ & $21 \pm 4$ \\
\hline Fiel 3 & - & $51 \pm 12$ & $29 \pm 16$ & $897 \pm 784$ & - & $66 \pm 102$ & $244 \pm 373$ & $103 \pm 24$ & $10 \pm 1$ & $3.2 \pm 1.0$ & $28 \pm 37$ & $10 \pm 1$ \\
\hline Fiel 10 & - & $78 \pm 19$ & $28 \pm 6$ & $650 \pm 134$ & - & $18 \pm 5$ & $80 \pm 9$ & $137 \pm 15$ & $5 \pm 1$ & $3.0 \pm 0.9$ & $17 \pm 1$ & $12 \pm 1$ \\
\hline Fiel 15 & - & $32 \pm 6$ & $21 \pm 4$ & $410 \pm 59$ & - & $16 \pm 3$ & $75 \pm 13$ & $86 \pm 15$ & $6 \pm 1$ & $2.9 \pm 0.4$ & $10 \pm 4$ & $8 \pm 2$ \\
\hline Fiel 30 & - & - & $39 \pm 5$ & $1,238 \pm 249$ & $9 \pm 2$ & $28 \pm 1$ & $135 \pm 20$ & $157 \pm 19$ & $6 \pm 1$ & $4.0 \pm 0.6$ & $20 \pm 3$ & $17 \pm 2$ \\
\hline Bism 3.4 & - & - & $25 \pm 3$ & $756 \pm 42$ & 2 & $10 \pm 2$ & $55 \pm 10$ & $100 \pm 12$ & $8 \pm 1$ & $3.1 \pm 0.5$ & $16 \pm 2$ & $14 \pm 2$ \\
\hline Bism 3.4 & - & - & 29 & 416 & 1 & 4 & 24 & 151 & 9 & 2.4 & 9 & 18 \\
\hline Bism 3.4 & - & - & $31 \pm 4$ & $1,125 \pm 189$ & 3 & $19 \pm 12$ & $33 \pm 25$ & $132 \pm 22$ & $9 \pm 1$ & $3.9 \pm 0.9$ & $15 \pm 2$ & $23 \pm 5$ \\
\hline Bism 3.3A (a) & - & $<330$ & $14 \pm 1$ & $581 \pm 27$ & 3 & $6 \pm 2$ & $35 \pm 4$ & $97 \pm 13$ & $5 \pm 1$ & $2.7 \pm 0.6$ & $12 \pm 4$ & $14 \pm 2$ \\
\hline \multicolumn{13}{|l|}{ Fluid inclusions } \\
\hline Bism 3.3A (b) & - & $<4,441$ & 30 & $168 \pm 18$ & $<45$ & $<7$ & $15 \pm 3$ & $1,010 \pm 516$ & $105 \pm 3$ & $85 \pm 39$ & $3 \pm 2$ & $3.0 \pm 2.0$ \\
\hline Bism 3.4B & - & $1,655 \pm 151$ & $118 \pm 10$ & $12,483 \pm 277$ & $38 \pm 4$ & $2 \pm 0.4$ & $9 \pm 3$ & $1,415 \pm 350$ & $3,723 \pm 48$ & $78 \pm 6$ & $4 \pm 3$ & $4.3 \pm 3.1$ \\
\hline Bism 3.3A & - & 1,304 & 260 & 7,662 & 136 & 6 & 17 & 1,335 & 3,165 & 427 & 4 & 2.5 \\
\hline EFlag2 & - & - & 127 & 2,216 & 80 & 295 & 16 & 180 & 2,857 & 74 & - & - \\
\hline Yank 20C & - & $586 \pm 161$ & $105 \pm 15$ & $3,115 \pm 401$ & - & - & $0.9 \pm 0.2$ & $37 \pm 12$ & $2,604 \pm 567$ & $6 \pm 2$ & - & - \\
\hline Yank 20C & - & $137 \pm 5$ & $49 \pm 13$ & $382 \pm 90$ & - & - & $0.8 \pm 0.2$ & $2.5 \pm 0.6$ & $324 \pm 5$ & $2 \pm 1$ & & \\
\hline Low w9 & $10 \pm 2$ & $360 \pm 121$ & $200 \pm 46$ & $3,702 \pm 864$ & - & $309 \pm 137$ & $0.8 \pm 0.9$ & $60 \pm 13$ & $7,937 \pm 2,165$ & $17 \pm 9$ & - & $0.4 \pm 0.4$ \\
\hline Low w9 & - & $76 \pm 36$ & $30 \pm 12$ & $39 \pm 20$ & - & $322 \pm 190$ & $0.4 \pm 0.5$ & $1.4 \pm 0.6$ & $171 \pm 94$ & $3 \pm 1$ & - & $0.3 \pm 0.2$ \\
\hline Wolf 12 & - & 53 & $149 \pm 1$ & $2,182 \pm 497$ & $169 \pm 12$ & 55 & - & $30 \pm 20$ & $3,443 \pm 1,085$ & $30 \pm 11$ & - & - \\
\hline Wolf 12 & - & - & $49 \pm 28$ & $128 \pm 16$ & $12 \pm 5$ & $378 \pm 180$ & - & - & $194 \pm 27$ & $1 \pm 0.4$ & - & - \\
\hline
\end{tabular}




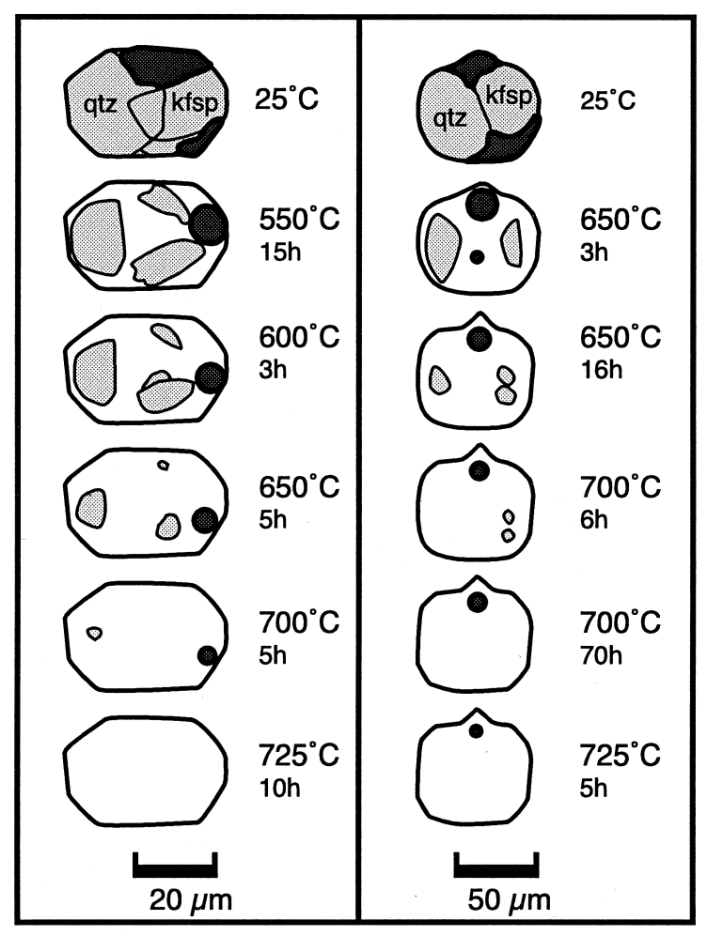

Figure 3. Melting sequence of crystallized melt inclusions in two chips of the same topaz sample. In the inclusion on the right, three hours residence time at $650{ }^{\circ} \mathrm{C}$ apparently was not enough to attain crystal-melt equilibrium, similarly for six hours residence time at $700{ }^{\circ} \mathrm{C}$.

The prototype system at ETH Zürich uses an 193 nm Excimer Laser (Lambda Physics, Germany) and special homogenization optics to create a laterally homogeneous laser energy distribution on the sample spot, permitting flat-bottomed holes of various size $(4,10,20,40,60$ and $80 \mu \mathrm{m})$ to be drilled even into problematic minerals like fluorite and certain low-absorbing quartz samples. The fact that the pit size can be adjusted during the measurement allows a controlled ablation process, and ensures that the entire inclusion content is transported into the mass spectrometer. The resulting transient signals (Fig. 4) are integrated and then compared with signals from standard reference materials, in order to calculate relative element abundances within the inclusions. To transform these relative concentrations into absolute values, the concentration of one element in the inclusion has to be determined prior to LA-ICP-MS by an independent method. In the case of fluid inclusions we use $\mathrm{Na}$ from the microthermometrically determined $\mathrm{NaCl}_{\text {equiv }}$-value, which we correct for other major cations present in the aqueous solution (Günther et al., 1998; Audétat et. al., 1999).

The standardization of melt inclusions is based on either $\mathrm{Al}$ or $\mathrm{K}$, depending on the type of host mineral. For melt inclusions in quartz we used the average Al-content of the whole-rock because this value varied only little during melt fractionation, and because $\mathrm{Al}$ is relatively insoluble compared to $\mathrm{K}$ and $\mathrm{Na}$ (the latter being partly remobilized from the quartz-hosted melt inclusions - see section 4.3). The compositions of melt inclusions hosted by topaz were calculated on the basis of K-concentrations determined by electron microprobe analysis in 13 re-homogenized melt inclusions. The same inclusions were also analyzed for $\mathrm{Na}, \mathrm{F}, \mathrm{Cl}, \mathrm{Si}, \mathrm{Al}, \mathrm{Fe}$ and $\mathrm{O}$. EPMA analyses were performed on a CAMECA SX50, using $15 \mathrm{kV} / 20 \mathrm{nA}$ and an expanded beam size to reduce fractionation effects. Fluorine was measured on a multilayer crystal.

The uncertainty introduced by the internal standardization procedure in crystallized melt inclusions is in the order of $20-30 \%$. This may appear to be rather high at first glance, but is actually acceptable when compared to the enormous concentration changes during the melt fractionation, with certain elements being enriched by more than two orders of magnitude. 


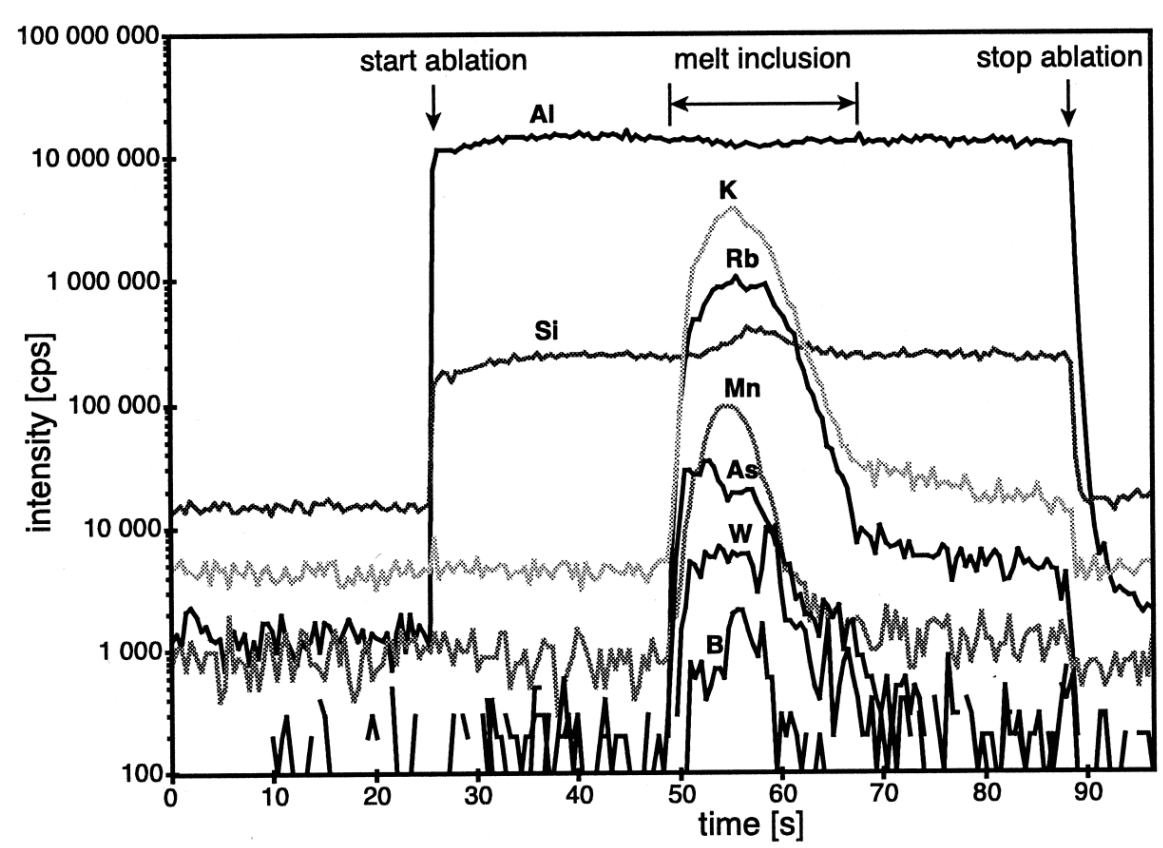

Figure 4. LA-ICP-MS signal of a crystallized melt inclusion in magmatic topaz, ablated through the Al-and Si-containing host mineral. The inclusion measured $\sim 40 \mu \mathrm{m}$ in diameter, and was ablated using a $60 \mu \mathrm{m}$ beam.

\section{Results}

\subsection{Ore composition versus fluid composition}

Data on the hydrothermal stage in veins and greisens of the Mole Granite are presented by Eadington (1983), Rankin et al. (1992), Audétat et al. (1998, 1999) and Audétat (1999). Detailed reconstruction of the fluid-chemical evolution in a typical Sn-dominated deposit (Yankee Lode; Audétat et al., 1998) and otherwise similar deposits dominated by W (Flagstone Lodes, Bismuth; Audétat et al. 1999) showed that the input fluids forming W-deposits are characterized by about 10 times higher W/Sn ratios than those of Sn-deposits. Ore mineral precipitation in both types of deposit was driven by the first dilution of the magmatic brine by cooler low-salinity water of probable meteoric origin. The occurrence of most major orebodies at the intersection of two veins (Mulholland, 1943) also indicates a common physical process that causes mineral precipitation. This result implies that the $\mathrm{W} / \mathrm{Sn}$-ratio in the ore deposit was controlled mainly by the initial W/Sn-ratio in the mineralizing fluid, and only to a lesser degree by selective metal precipitation. The type of ore deposit (Sn vs. W-dominated) was therefore a function of processes operating closer to the magmatic source of the fluids, prior to their focusing into the ore veins.

\subsection{Composition of granitic magmas and residual melts}

Key information about the magmatic-hydrothermal transition stage has been gained from LAICP-MS analysis of crystallized melt inclusions (Table 1) and their comparison with the bulk composition of the Mole Granite. Figure 5 shows that the composition of the melt inclusions is much more variable than that of the three textural variants of the bulk granite.

The composition of the melt inclusions follows a systematic fractionation trend that seems to reflect an increasing degree of crystallization and geochemical specialization of the residual melts. The oldest melt inclusions are found on early growth zones within quartz phenocrysts (Fig. 6), 
implying that they were trapped at a very low degree of crystallinity (probably less than 10\%). Their composition is very similar to that of the average Mole Granite, with the exception of B, W, $\mathrm{Sb}$ and As which occur at distinctly higher concentrations in the melt inclusions. Slightly younger melt inclusions occur in matrix-quartz of the seriate granite, showing a further enrichment in all incompatible elements (Cs, B, Sn, W, Sb, As) and relative depletion in most compatible elements. The latter can be explained by the crystallization of plagioclase $(\mathrm{Sr}, \mathrm{Ba})$, biotite $(\mathrm{Fe}, \mathrm{Mg}, \mathrm{Ti}$ ), ilmenite (Fe, Ti), monazite (La, Ce), zircon, and thorite (Zr, U, Th). Very late generations of melt inclusions occur both in magmatic topaz and in embayment structures of quartz phenocrysts. Typical melt inclusions in topaz contain 2-3 wt\% F, 4000 ppm Cs, 2800 ppm Cl, 1000 ppm B, 700 ppm As and $140 \mathrm{ppm} \mathrm{W} \mathrm{(Table} \mathrm{1),} \mathrm{corresponding} \mathrm{to} \mathrm{enrichment} \mathrm{factors} \mathrm{of} \mathrm{20-300} \mathrm{relative} \mathrm{to} \mathrm{the}$ average composition of the Mole Granite. Similar enrichment factors are reported for melt inclusions in pegmatitic quartz from a Sn/W-mineralized granite in the Central Erzgebirge, Germany (Webster et al., 1997).

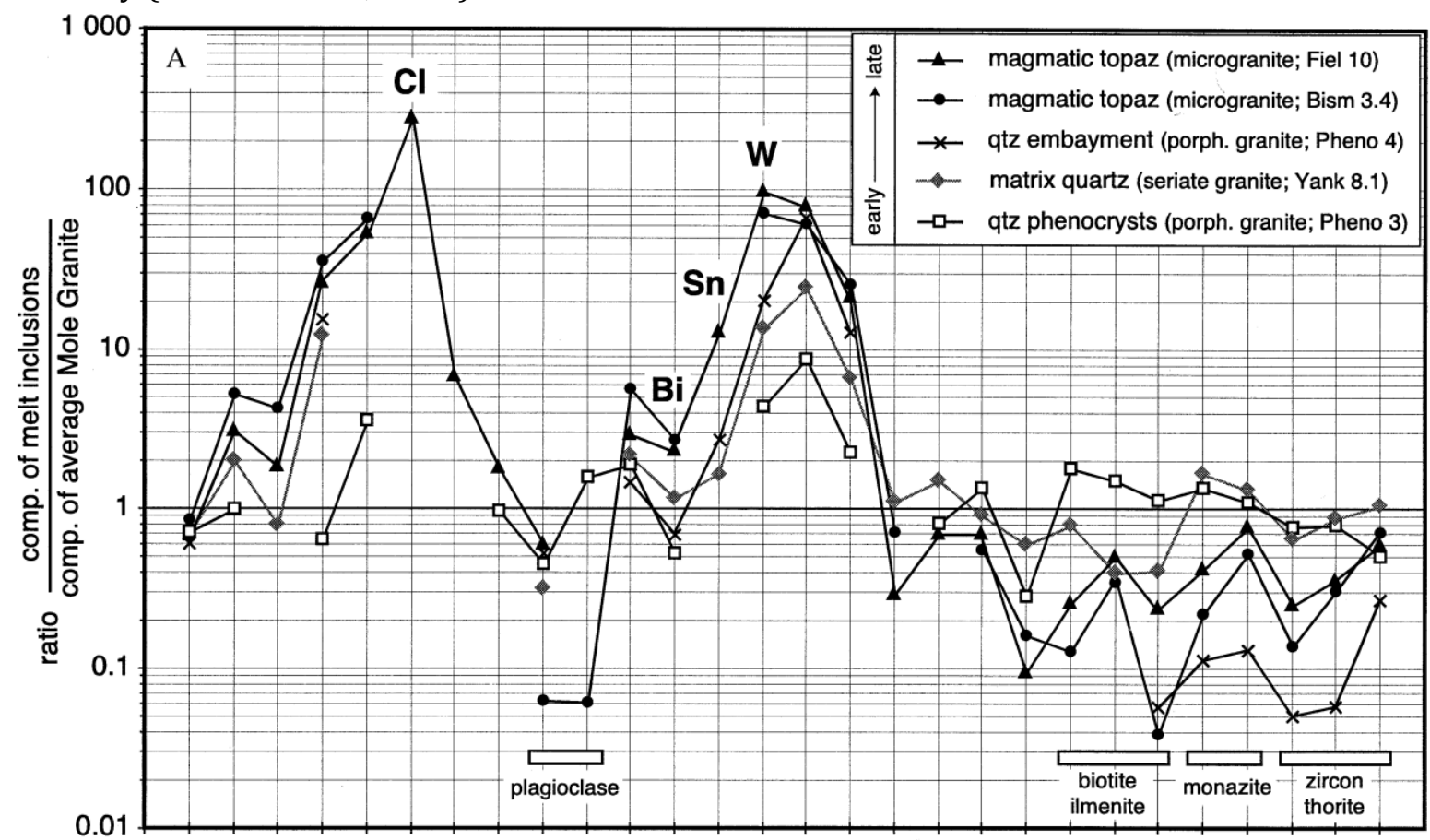

$\mathrm{K}$ Rb Li Cs B Cl F P Sr Ba Mn Bi Sn W Sb As Cu Ag Zn Pb Ti Fe Mg La Ce Zr Th U

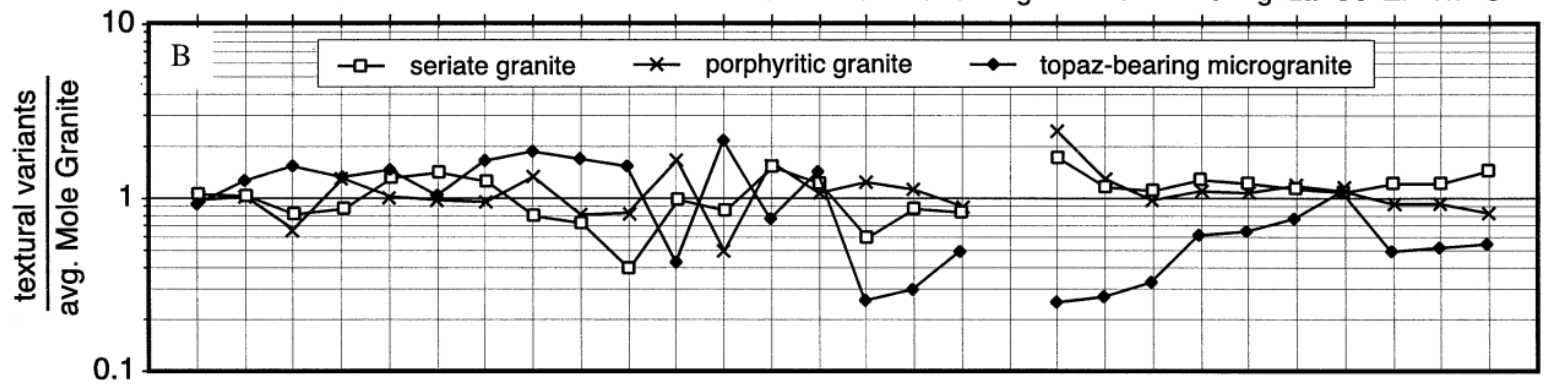

Figure 5. (a) Composition of residual melts at various degrees of crystallization and geochemical fractionation, reconstructed from electron microprobe (F, Cl, P) and LA-ICP-MS (all other elements) analysis of different generations of melt inclusions in quartz phenocrysts and magmatic topaz. To emphasize elemental fractionation trends the absolute element concentrations were normalized to the average whole rock composition of the Mole Granite (Table 1). Incompatible elements display positive fractionation trends, whereas compatible elements (incorporated in the minerals shown at the bottom) display negative fractionation trends. (b) Variation of the whole rock composition of the three textural variants, plotted onto the same scale that is used in Fig. 5a (see text for further explanations). 
Cathodoluminescence images (Fig. 6) show that the embayment structures in quartz phenocrysts are a primary growth phenomenon, and not the result of quartz resorption. Similar evidence has been presented also by Laemmlein (1930), Anderson (1991) and Lowenstern (1995). Parts of these embayment structures were subsequently filled with late-stage quartz, leading to the entrapment of melt, vapor, brine and coexisting solids in the quartz phenocryst.

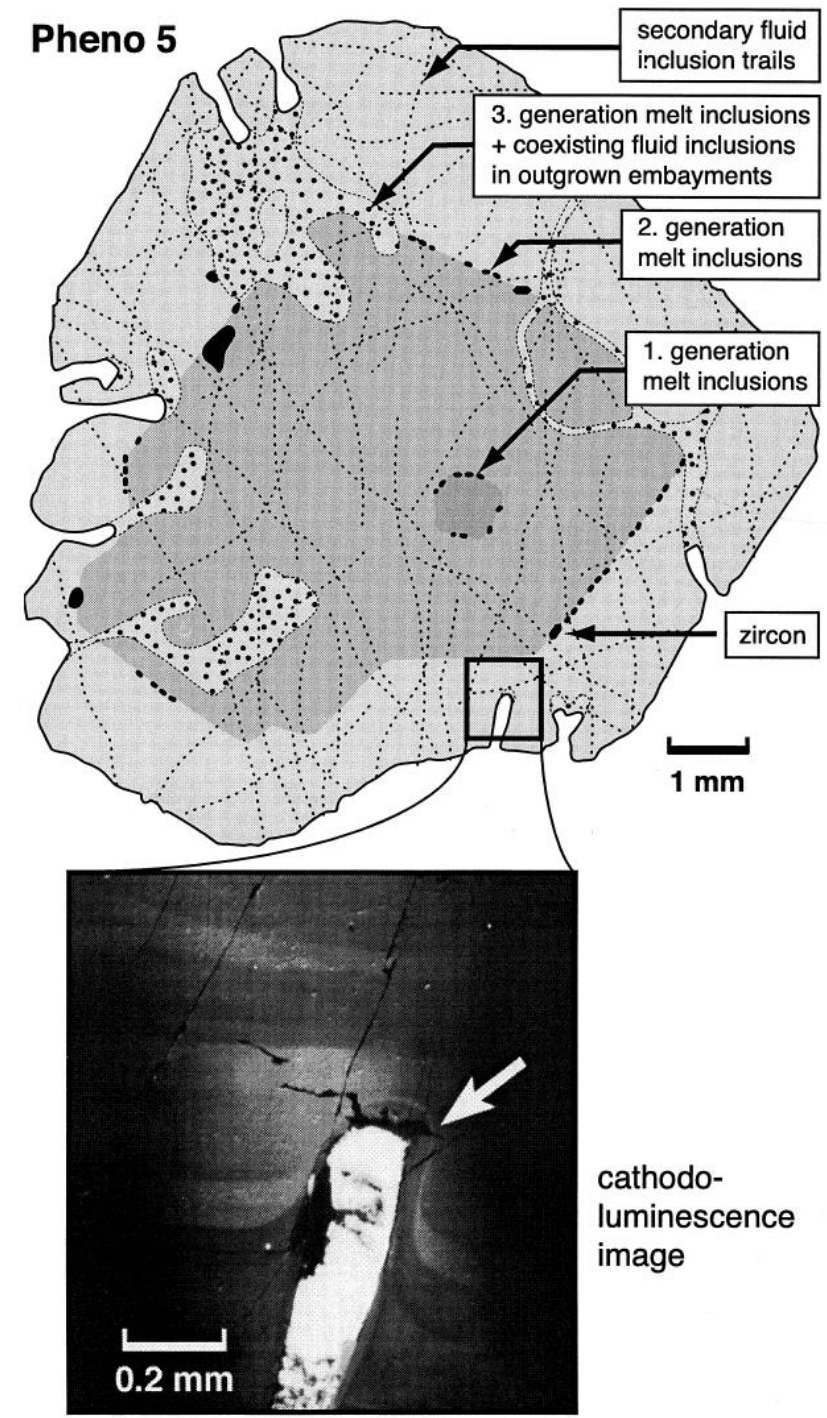

Figure 6. Schematic view of the internal structure of quartz phenocrysts found in the Mole Granite, interpreted on the basis of cathodoluminescence images. The fact that the growth zones bend into the embayment structures (white arrow) proves that these structures are a primary growth feature, and not the result of resorption.

One of the most difficult tasks in the reconstruction of a magmatic-hydrothermal evolution is to determine the moment at which fluid saturation was attained in the melt, and to find out the state of the exsolving fluid (i.e., whether it was two-phase or single-phase). The occurrence of a free fluid phase in the Mole Granite is first documented by the development of sheeted vein systems in the overlying country rocks. The fact that these sheeted veins are cut and displaced by microgranite indicates that they have formed before the intrusion of the microgranite, probably during the formation of the porphyritic carapace. It is unlikely that fluid-saturation was attained much earlier, as early melt inclusions in quartz phenocrysts never are associated with fluid inclusions, and because if fluid saturation was attained earlier it probably would have led to 
explosive magmatism or granite emplacement at greater depth. Note that the small grain size in the matrix of porphyritic granite is not necessarily the product of pressure-quenching, but could simply be the result of crystallization under water-saturated conditions (Whitney, 1988). In either case it implies that the melt was fluid-saturated at that stage. As the microgranite formed later, it must have been fluid-saturated during its whole crystallization history. This is confirmed by the melting behavior of the crystallized melt inclusions in topaz, which usually attain complete homogenization by simultaneous disappearance of the vapor bubble and daughter minerals within a range of a few tens of degrees (Fig. 2e and Fig. 3).

\subsection{Coexisting melt and fluid}

Some magmatic topaz crystals contain melt inclusions that undoubtedly coexisted with an aqueous fluid. Such fluid - melt assemblages were observed both on primary growth zones as well as on pseudosecondary cracks similar to pseudosecondary trails of fluid inclusion (Fig. 7).

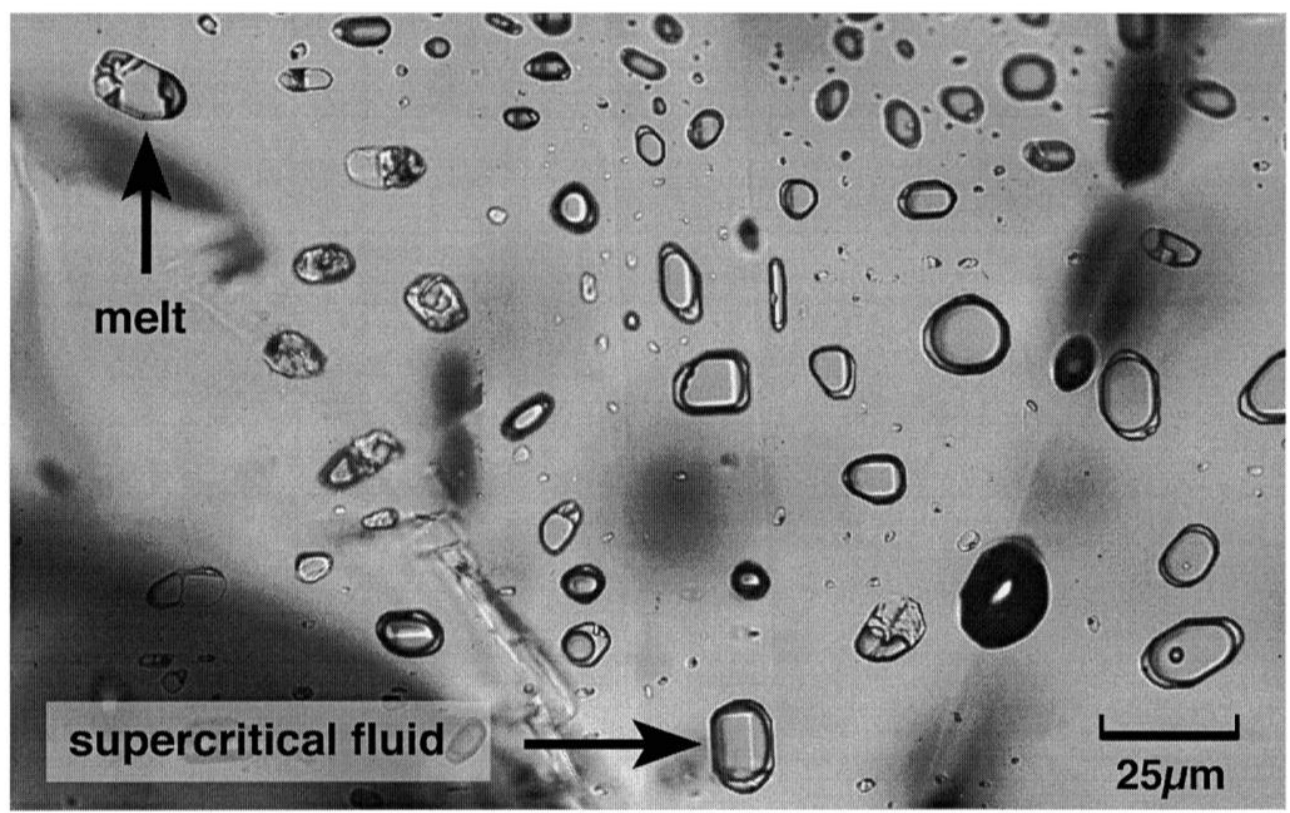

Figure 7. Photomicrograph of a pseudosecondary trail in magmatic topaz, showing crystallized melt droplets which unambiguously coexisted with a single-phase fluid of low density and salinity. Transmitted light.

Due to the similar appearance of crystallized melt inclusions and highly saline brine inclusions at room temperature the positive identification of melt inclusions sometimes was possible only by heating experiments (earlier first melting in brine inclusions; glass formation during quench of melt inclusions) or by LA-ICP-MS analysis (lower Cl-content in the melt inclusions compared to brine inclusions). Fluid inclusions coexisting with melt inclusions in topaz are of low density $(\sim 0.2$ $\mathrm{g} / \mathrm{cm}^{3}$ ) and moderately low salinity (3.5-6.5 $\mathrm{wt} \% \mathrm{NaCl}_{\text {equiv. }}$ ), and seem to have been trapped in the single-phase region of the salt - water system. The latter is indicated by the general absence of brine inclusions, despite the fact that their wetting properties would render an entrapment more likely. Supporting evidence is given also by their composition, which is very unusual compared to that of vapor inclusions in true boiling assemblages. Vapor inclusions coexisting with brine inclusions are characterized by high $\mathrm{Cu}$-contents (usually above $2000 \mathrm{ppm}$ ) and $\mathrm{W}$-, Bi-, and $\mathrm{Zr}$ concentrations well below 5 ppm (Table 1; see also Heinrich et al., 1992; Audétat et al. 1998, Audétat et al., 1999, Heinrich et al., 1999, and Audétat, 1999). In contrast to this the low-density fluid inclusions coexisting with melt inclusions in topaz contain little $\mathrm{Cu}(<400 \mathrm{ppm})$, but high 
concentrations of $\mathrm{W}(\sim 1000 \mathrm{ppm}), \mathrm{Bi}(85 \mathrm{ppm})$ and $\operatorname{Zr}(18 \mathrm{ppm})$ (i.e., even more than most brine inclusions; see Table 1). Analyses of melt inclusions and coexisting fluid inclusions trapped on a pseudosecondary trail similar to that shown in Fig. 7 allowed direct determination of partition coefficients for 11 elements $D_{\text {(supercrit.fluid) } / \text { melt }}=c_{x, \text { fluid }} / c_{x}$, melt (Fig. 8).

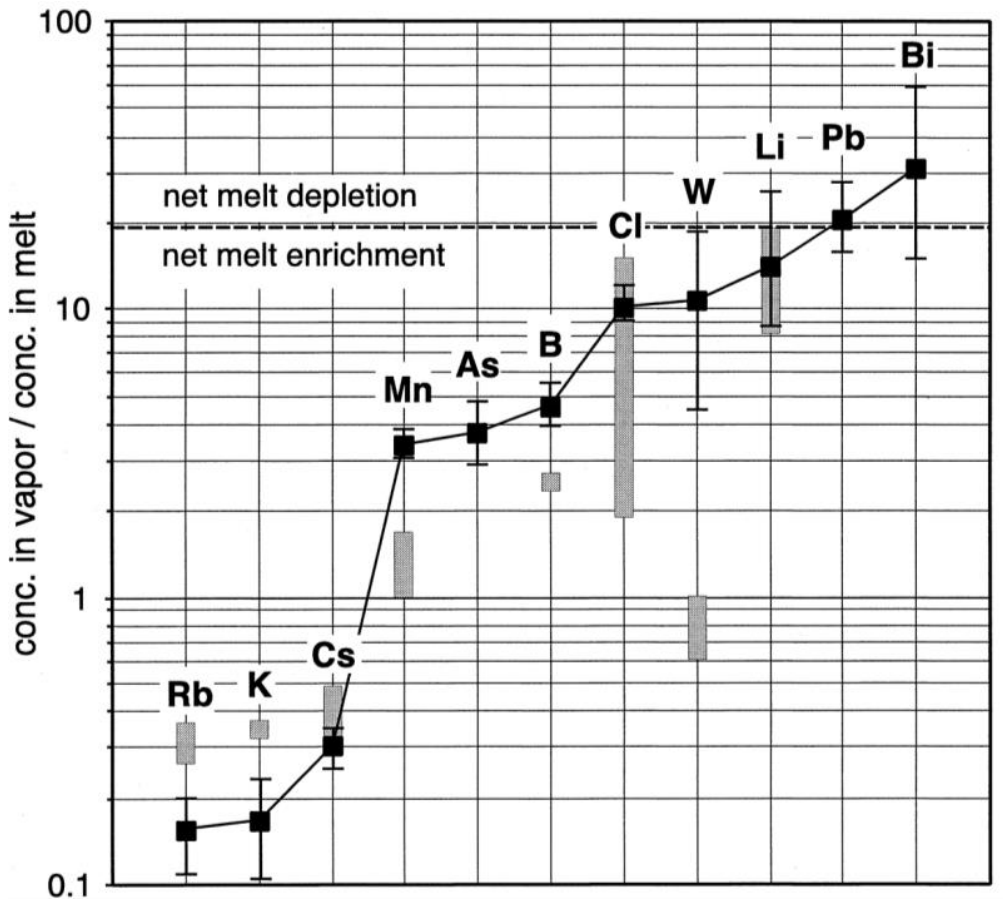

Figure 8. Fluid - melt partition coefficients (Dfluid/melt) of 11 elements, calculated from LA-ICP-MS analyses of crystallized melt inclusions and fluid inclusions on a trail similar to that in Fig. 7. Grey bars show experimentally determined fluid melt partition coefficients compiled by Candela and Piccoli (1995). Note that a D fluid/melt -value of $>1$ does not necessarily mean that the corresponding element becomes depleted in the crystallizing melt. In fact, this is true only if the fluid/meltratio is greater than 1 . Normal granitic melts, however, attain fluid saturation at $\sim 5 \mathrm{wt} \% \mathrm{H}_{2} \mathrm{O}$ (i.e., a fluid/melt-ratio of 0.05), where $D_{x}$, fluid/melt has to be $>19$ to result in a depletion of the element $X$ in the melt (assuming $100 \%$ incompatibility of $\mathrm{X}$ and $\mathrm{H}_{2} \mathrm{O}$ with respect to the crystallizing minerals).

Most of the measured elements clearly fractionate into the single-phase aqueous fluid, except for $\mathrm{Rb}, \mathrm{K}$ and $\mathrm{Cs}$ which preferentially fractionate into the melt. This general trend agrees well with data presented in Candela and Piccoli (1995), who compiled a large number of experimental studies. The only element that shows a major discrepancy is $\mathrm{W}$, which probably is due to the fact that the corresponding experiments were performed with solutions that contained no chlorine.

In the very last stages of crystallization two separate fluid phases seem to have exsolved from the microgranitic melts, as indicated by the coexistence of melt and brine inclusions on a late growth zone within magmatic topaz, and the presence of vapor inclusions on pseudosecondary trails of approximately the same age. Brine inclusions coexisting with this last generation of melt have a salinity of $37-40 \mathrm{wt} \% \mathrm{NaCl}_{\text {equiv. }}$ and homogenize by bubble disappearance at temperatures between $620^{\circ} \mathrm{C}$ and $670{ }^{\circ} \mathrm{C}$, whereas vapor inclusions occurring on adjacent trails show average salinities of $4 \mathrm{wt} \% \mathrm{NaCl}_{\text {equiv. }}$ Inclusions that trapped both melt and fluid ("heterogeneous trapping") show a very complex heating behavior, with two separate silicate (?) melts and one fluid phase coexisiting at some stage. Primary brine inclusions in later, metasomatic topaz (i.e., in the quartz-topaz greisens) homogenize at $500-560{ }^{\circ} \mathrm{C}$, have salinities of $34 \pm 4 \mathrm{wt} \% \mathrm{NaCl}_{\text {equiv., }}$ and coexist with vapor inclusions of 3.7-5.4 wt\% $\mathrm{NaCl}_{\text {equiv. }}$ salinity.

The melts of the porphyritic granite followed a slightly different path of magmatichydrothermal evolution. Coexistence with single-phase fluids could not be observed in these rocks. 
Instead, the melt inclusions in the embayment structures of quartz phenocrysts always coexist with both vapor and brine inclusions. The salinity of the brine inclusions is comparatively low (27$\left.30 \mathrm{wt} \% \mathrm{NaCl}_{\text {equiv. }}\right)$, whereas the salinity of the coexisting vapor inclusions is relatively high (7.5-9 $\mathrm{wt} \% \mathrm{NaCl}_{\text {equiv. }}$, very similar to the salinities of high-pressure boiling assemblages found in quartz crystals from the Yankee Lode (Audétat et al., 1998). However, the brine inclusions homogenize already at $360-380{ }^{\circ} \mathrm{C}$, when coexisting vapor inclusions still are clearly two-phase. This indicates that these inclusions were subject to significant post-entrapment modifications, probably involving substantial loss of water (Audétat and Günther, 1999).

Water appears to have been lost also from certain melt inclusions (particularly those hosted by quartz), resulting in unreasonably high melting temperatures $\left(>1000{ }^{\circ} \mathrm{C}\right)$ and extremely low Nacontents. Instead of an expected value of $\sim 2.5 \mathrm{wt} \% \mathrm{Na}$ these inclusions contain only 1000-2000 ppm $\mathrm{Na}$, whereas most other elements occur in geologically reasonable concentrations. Melt inclusions hosted by topaz on the other hand reveal both "normal" Na-concentrations (2.0-2.5 $\mathrm{wt} \%)$, as well as extremely deficient values (1000-5000 ppm), with no notable changes in all other elements. The fact that Na-deficiencies are less common in the more resistant topaz suggests that they are somehow related to deformation processes. However, it is not clear why only Na was lost in such substantial amounts, whereas other elements like $\mathrm{K}, \mathrm{Li}, \mathrm{Rb}, \mathrm{Mn}$ and Fe remained in basically unchanged concentrations within the melt inclusions. Thomas et al. (1996) and Webster et al. (1997), who performed EPMA on re-homogenized melt inclusions from the Erzgebirge (SE Germany), do not report suspiciously low Na-values. This may be explained by the fact that they automatically chose melt inclusions that retained their volatiles, since all other inclusions would not homogenize (or if so, only at much higher temperatures). See Lowenstern (1995) for a general discussion about the validity of compositions preserved in melt inclusions.

\section{Discussion}

\subsection{The importance of fluorine}

Evidence reported in the previous section demonstrates that the Sn/W-ratio in the ore deposits was primarily a function of the $\mathrm{Sn} / \mathrm{W}$-ratio in the magmatic input fluid. The attention was therefore addressed to the question: What controls the composition of a magmatic fluid? Basically, the composition of an aqueous fluid in equilibrium with melt is determined by the fluid-melt partition coefficients $\left(\mathrm{D}_{\text {fluid/melt }}\right)$, which in turn are a function of pressure $(\mathrm{P})$, temperature $(\mathrm{T})$, and melt composition (X). In the case of the Mole Granite there is no evidence that the W-rich fluids exsolved at considerably different $\mathrm{P} / \mathrm{T}$-conditions than the Sn-rich fluids, suggesting that the variable Sn/Wratio is caused by differences in the melt composition (X). The LA-ICP-MS analyses plotted in Fig. 5 a demonstrate that the absolute concentration of $\mathrm{Sn}$ and $\mathrm{W}$ in the residual melts strongly increased with progressive fractionation, but that the ratio of $\mathrm{W} / \mathrm{Sn}$ remained about the same (note that the original melt composition is more likely to be represented by the first generation of melt inclusions than by the whole-rock, as the latter is expected to be depleted in all fluidfractionating elements). The highly variable $\mathrm{W} / \mathrm{Sn}$-ratio in the magmatic fluid is therefore not a reflection of the W/Sn-ratio in the melt, but apparently the result of other melt components that indirectly controlled the fluid - melt partition coefficients ( $\left.D_{\text {fluid/melt }}\right)$ of $S n$ and W. In the following discussion we will show that the main controlling element was probably fluorine.

Even if the underlying mechanisms are still debated it is generally accepted that $F$ (and probably $\mathrm{H}_{2} \mathrm{O}$ ) tend to accumulate in the apical portions of crystallizing magma chambers (Hildreth, 1981; MacKenzie et al., 1988; Skirius et al., 1990; Dunbar and Hervig, 1992; Webster and Duffield, 1994; Dostal and Chatterjee, 1995, Wallace et al., 1999). Evidence for such a 
compositional gradient in the Mole Granite is found in: (i) the fact that the topaz content of the rock increases from seriate granite over porphyritic granite to microgranite, (ii) the upward enrichment of magmatic topaz in microgranitic sills, and (iii) the accumulation of phenocrysts and mafic enclaves in the lower part of the porphyritic carapace, implying a density- and/or viscosity gradient in the melt (Stegmann, 1983; and own observations).

Experimental data demonstrate that the presence of fluorine has a strong influence on the subsequent history of crystallization and fluid exsolution, which partly is due to the influence of fluorine on Cl-partitioning between fluids and silicate melts (Webster and Holloway, 1990).

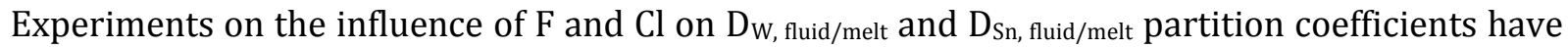
lead to conflicting results with respect to both qualitative and quantitative behavior (Manning and Henderson, 1984; Taylor and Wall, 1984; Wood and Vlassopoulos, 1989; Keppler and Wyllie, 1991; Zilong and Shenglin, 1994; Candela and Piccoli, 1995). These discrepancies can be explained by the fact that $\mathrm{F}$ and $\mathrm{Cl}$ were added in some experiments as $\mathrm{HF}$ and $\mathrm{HCl}$ (Keppler and Wyllie, 1991), and in others as alkali salts like $\mathrm{NaF}$ and $\mathrm{NaCl}$ (Manning and Henderson, 1984; Taylor and Wall, 1984; Zilong and Shenglin, 1994). At the high fluid/melt ratios commonly used for these experiments the addition of $\mathrm{F}$ and $\mathrm{Cl}$ in form of alkali-salts changes the alkali/alumina-ratio in the melt, leading to dramatic changes in the $\mathrm{D}_{\text {fluid/melt }}$ partition-coefficients (Urabe, 1985). For this reason we regard the experiments performed with HF and $\mathrm{HCl}$ (Wood and Vlassopoulos, 1989; Keppler and Wyllie, 1991) as more applicable for our quantitative modelling. The following experimental results have direct implications on the magmatic-hydrothermal evolution of F-rich melts:

1) The addition of fluorine to a melt leads to an exponential reduction of its viscosity. This effect is most pronounced with the addition of the first 1-2 wt \% of fluorine (Dingwell et al., 1993; Lange, 1994).

2) Fluorine has no major influence on the solubility of $\mathrm{H}_{2} \mathrm{O}$ in the melt, but strongly enhances the solubility of silicates in the fluid (London, 1987; Webster and Holloway, 1990).

3) $\mathrm{D}_{\mathrm{Cl} \text {, fluid/melt }}$ decreases logarithmically with increasing F-content of the melt (Webster and Holloway, 1990). The value for $\mathrm{D}_{\mathrm{Cl}}$, fluid/melt decreases from about 25 in fluorine-free melts to less than 6 at 3 wt $\% \mathrm{~F}$.

4) $D_{F}$, fluid/melt on the other hand increases logarithmically with increasing F-concentration in the melt (Webster and Holloway, 1990). However, even at high fluorine concentrations in the

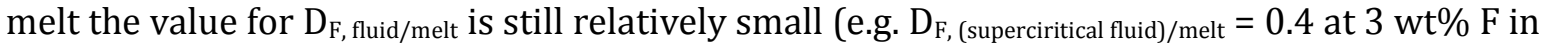
the melt), suggesting that the exsolution of fluid will not lead to fluorine depletion of the melt.

5) $\mathrm{D}_{\text {Cl fluid/melt }}$ increases linearly with increasing Cl-content of the melt, until the latter reaches a maximum at $\sim 3500 \mathrm{ppm} \mathrm{Cl}$. At this stage, $\mathrm{D}_{\mathrm{Cl} \text { fluid/melt }}$ suddenly shifts to much higher values (Webster and Holloway, 1990).

6) $D_{S n \text {, fluid/melt }}$ and $D_{W}$, fluid/melt are largely independent of the fluorine-content of the system, or slightly decrease with increasing F-concentration (Manning and Henderson, 1984; Keppler and Wyllie, 1991; Zilong and Shenglin, 1994).

7) $\mathrm{D}_{\mathrm{W} \text {, fluid/melt }}$ and $\mathrm{D}_{\mathrm{W}, \text { wo3/fluid }}$ remain unchanged if $\mathrm{Cl}$ is added to the system (Wood and Vlassopoulos, 1989; Keppler and Wyllie, 1991), $\mathrm{D}_{\mathrm{W}, \text { fluid/melt }}$ being on the order of 1.

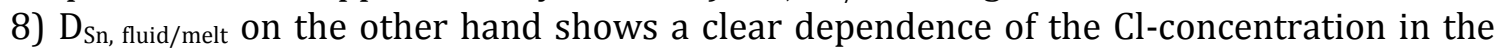

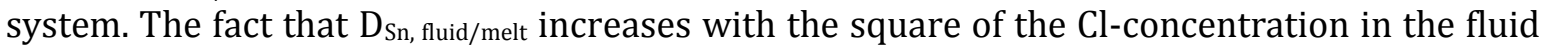
indicates that it is transported in form of $\mathrm{SnCl}_{2}$ (Taylor and Wall, 1984; Lehmann, 1990; Keppler and Wyllie, 1991).

Combination of points 3, 4, 5, 6, 7 and 8 forms the basis of a quantitative model that explains most features of the evolution of the Mole Granite. 


\section{2. $\quad$ Mass-transfer model based on experimental and microanalytical constraints}

We have modeled the behavior of $\mathrm{Cl}, \mathrm{F}, \mathrm{H}_{2} \mathrm{O}$, Sn and $\mathrm{W}$ in a crystallizing system that explains the observed evolution of melts and fluids in the Mole Granite. The calculations were made on the basis of a Rayleigh-type fractionation model (Fig. 9) similar to those used by Cline and Bodnar (1991), Shinohara (1994) and Candela and Piccoli (1995).

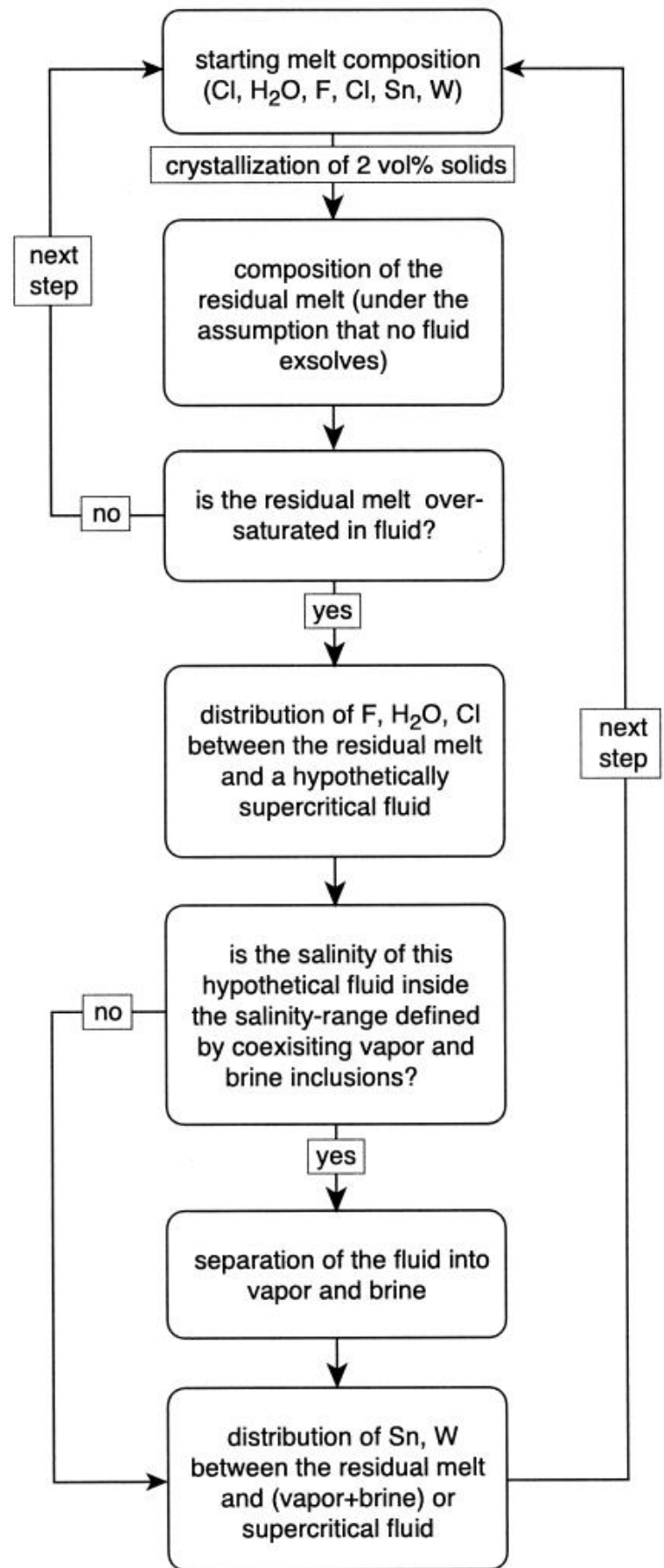

Figure 9. Simplified flow diagram of the Rayleigh fractionation model that was used to predict the composition of residual melts and exsolving fluids as a function of the crystallization degree. 
The following model assumptions and equation parameters were used in our model:

$-\mathrm{Cl}, \mathrm{H}_{2} \mathrm{O}, \mathrm{Sn}$ and $\mathrm{W}$ are assumed to be $100 \%$ incompatible with respect to the crystallizing minerals.

- Fluorine is assumed to behave $100 \%$ incompatible until the onset of topaz crystallization, at which point the F-concentration is assumed remain constant. Based on the fluorine content measured in re-homogenized melt inclusions in topaz (Table 1), this limit was set at $2.5 \mathrm{wt} \% \mathrm{~F}$. The assumption that fluorine behaved $100 \%$ incompatible before topaz crystallization is not entirely correct because it is incorporated also in biotite. However, the amount of biotite formed is relatively small ( $>5 \mathrm{vol} \%$ ), and its F-content 3-5 times lower than that of topaz.

- The melt is assumed to reach fluid saturation at a water-content of $4 \mathrm{wt} \% \mathrm{H}_{2} \mathrm{O}$. This value is based on experimental studies in the haplogranite system (Johannes and Holtz, 1996), and on petrologic relationships in the phase diagram of the Cape Ann Granite (Whitney, 1988).

- The Cl-solubility in the melt is assumed to reach a maximum at $3500 \mathrm{ppm}$, based on experiments performed by Carroll and Webster (1994).

- The effect of $\mathrm{F}$ and $\mathrm{Cl}$ on the fluid - melt partitioning coefficient of $\mathrm{Cl}$ has been expressed as $\mathrm{D}_{\mathrm{Cl} \text {, fluid } / \mathrm{melt}}=10^{\wedge}\left(\mathrm{q}-0.2023^{*} \mathrm{~F}_{\text {in melt }}[\mathrm{wt} \%]\right)+0.013^{*}\left(\mathrm{Cl}_{\text {in melt }}[\mathrm{ppm}]\right)$. The qualitative aspect of this equation is adopted from experimental results published in Webster and Holloway (1990), whereas q empirically was set at 1.6. In light of the complex but poorly known dependence of $q$ on additional factors like pressure and the $\mathrm{Al}_{2} \mathrm{O}_{3} /\left(\mathrm{CaO}+\mathrm{Na}_{2} \mathrm{O}+\mathrm{K}_{2} \mathrm{O}\right)$-ratio in the melt (Webster and Holloway, 1990) the use of an empirical q-value seems to be justified.

- The boundary of the two-phase field of the $\mathrm{NaCl}-\mathrm{H}_{2} \mathrm{O}$-dominated hydrothermal fluids was set at $5 \mathrm{wt} \% \mathrm{NaCl}_{\text {equiv. }}$ and $35 \mathrm{wt} \% \mathrm{NaCl}_{\text {equiv. }}$ for the low-salinity vapor and high-salinity brine, respectively, based on measured fluid inclusion salinities (Table 1).

- The influence of fluid salinity on the $\mathrm{D}_{\mathrm{Sn} \text {, fluid/melt }}$ partition coefficient has been expressed as $D_{\text {Sn, fluid/melt }}=10^{\wedge}\left(q+2 * \log \left(\mathrm{Cl}_{\text {in fluid }}[\mathrm{ppm}] / 35,450\right)\right.$. This equation is adopted from the results of Taylor and Wall (1984), with q empirically set at -0.8 .

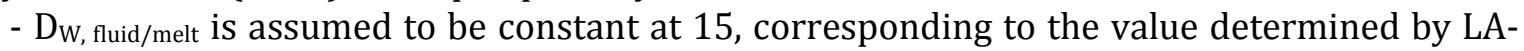
ICP-MS analysis of coexisting melt- and single-phase fluid inclusions in sample Bism 3.3 (Table 1).

Note that all these model assumptions are based on experimentally determined $\mathrm{D}_{\text {fluid/melt }}$ partition coefficients, and not on Na-normalized $K_{D}$ fluid/melt values as in the model of Candela (1992) and Candela and Piccoli (1995). The normalization to $\mathrm{Na}$ is not necessary in this case because the dependency of the fluid - melt partition coefficients on the chlorine concentration in melt and fluid is known, being explicitly incorporated in the model.

Figure 10 shows the compositional evolution of a crystallizing melt and exsolving fluids, predicted on the basis of one specific set of starting parameters. As long as the melt is not saturated in fluid (step I in Fig. 10a) the concentration of $\mathrm{Cl}, \mathrm{H}_{2} \mathrm{O}, \mathrm{F}, \mathrm{W}$ and $\mathrm{Sn}$ in the residual melt increases exponentially. Fluid saturation is attained at $40 \%$ crystallinity, at a stage at which the fluorine concentration in the melt is still relatively low. This results in relatively high $\mathrm{D}_{\mathrm{Cl}}$, fluid/melt-values, and in fluid salinities that lie within the salinity-range of the two-phase field (step II). As a consequence, the fluid separates into a vapor phase and a coexisting brine phase. The relatively high average salinity of the fluids leads to a preferential enrichment of Cl-complexed elements, and correspondingly smaller enrichment factors (or even depletion) of these elements in the crystallizing melt. This effect is further reinforced by the presence of the highly saline brine because the fluid - melt partition coefficient of a Cl-complexed element $\mathrm{XCl}_{n}$ increases with the $\mathrm{n}^{\text {th }}$ power of the $\mathrm{Cl}$-concentration in the fluid. For this reason, the fractionation of Cl-complexed elements is more effective into a boiling fluid assemblage than into a single-phase fluid of the same average salinity. $\mathrm{D}_{\mathrm{W}}$, fluid/melt on the other hand shows no dependence on the abundance of $\mathrm{Cl}$, finally leading to a low $\mathrm{W} / \mathrm{Sn}$-ratio in the fluid at stage II. 

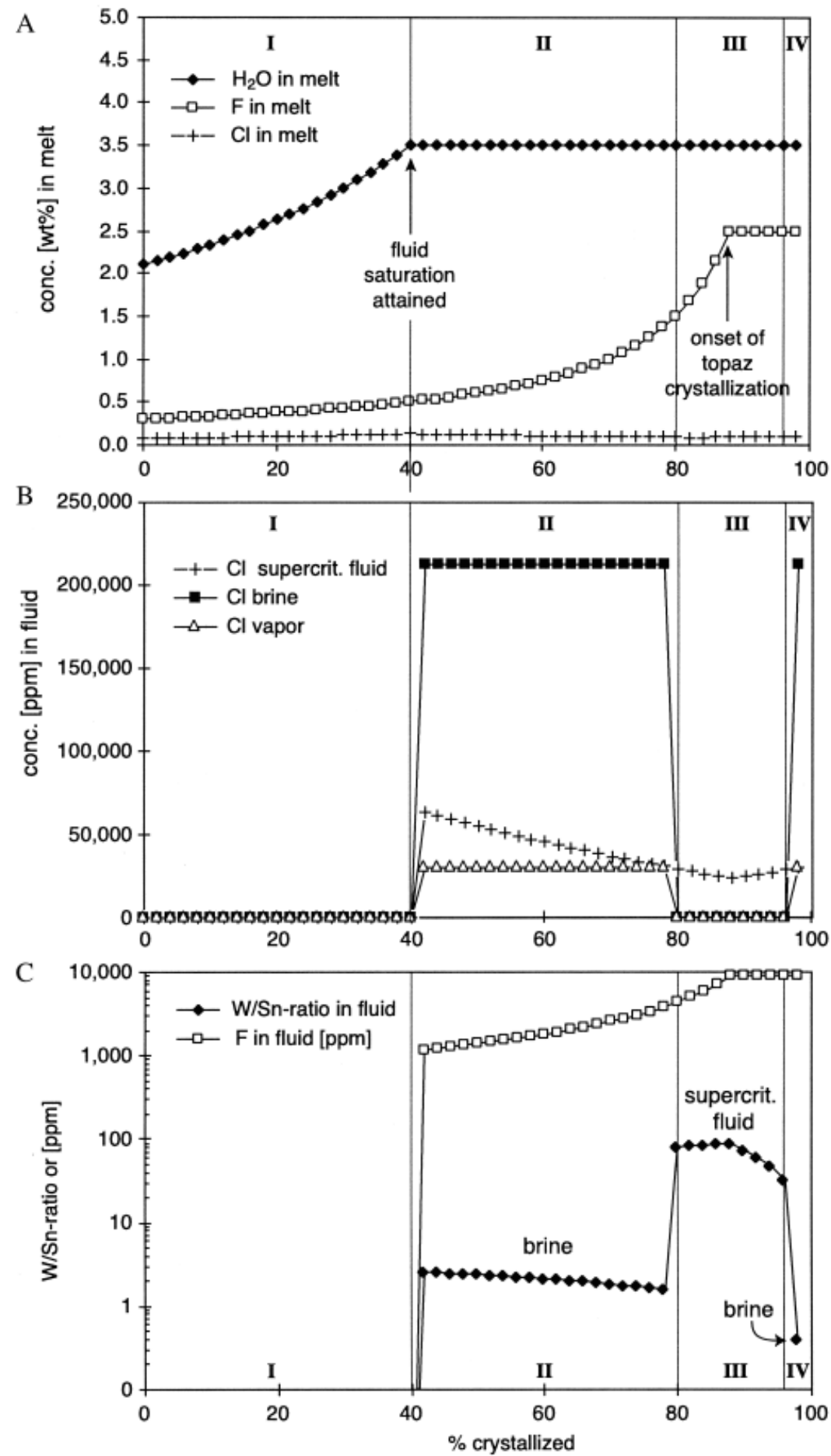

Figure 10. Prediction of the compositional evolution of melt and coexisting fluid, based on the Rayleigh fractionation model presented in Fig. 9. (a) The concentrations of H2O and F in the melt increase logarithmically until they are stabilized by the beginning of fluid exsolution (H2O) and the crystallization of topaz respectively. (b) The first exsolving fluid is relatively saline and therefore splits up into a vapor- and a coexisting brine phase (stage II). The increasing Fconcentration in the melt leads to a lower bulk salinity of the therefrom exsolving fluid, which finally lies outside the range of the two-phase field (stage III). As soon as the F-concentration of the melt is stabilized by the crystallization of topaz, DCl, fluid/melt starts to increase and causes the fluid to re-enter the two-phase field (stage IV). (c) The salinity directly controls the ore-metal content of the fluid, with low W/Sn-ratios prevailing in the highly saline brine inclusions and high W/Sn-ratios and F-concentrations occurring in the single-phase fluid of moderate salinity (the W/Sn ratio of the vapor phase is not plotted in this diagram). See text for further explanations. 
As $\mathrm{D}_{\mathrm{Cl}}$, fluid/melt decreases with increasing fluorine concentration in the residual melt the salinity of the exsolving fluid steadily decreases. At about $80 \%$ crystallization the bulk salinity starts to lie outside the salinity range of the two-phase field, resulting in the exsolution of a single-phase fluid

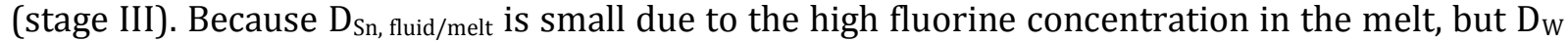
fluid/melt invariably high, this fluid is characterized by a high W/Sn-ratio and a relatively high content in fluorine (Fig. 10c). We believe that this fluid (or vapor - brine pairs derived from it during pressure drops) was responsible for the formation of the $\mathrm{W}$ deposits.

The situation changes at $\sim 90 \%$ crystallinity, where the fluorine-concentration in the melt is fixed by the crystallization of topaz (stage III). From this point, $\mathrm{D}_{\mathrm{Cl}}$, fluid/melt is not lowered further, and the salinity of the exsolving fluid increases again. At $~ 98 \%$ crystallinity the fluid therefore reenters the two-phase field (stage IV).

Note that the crystallization degrees in Fig. 10 reflect only the state of the bulk magma chamber. If residual melts separate from the site of crystallization and begin to accumulate at another place, their crystallization degree is reset to $0 \%$. In the case of the microgranitic melts this separation seems to have occurred at about $80 \%$ crystallinity because petrographic evidence suggest a coexistence with a single-phase fluid during most of their crystallization. Also, there apparently existed a vertical gradient of $\mathrm{H}_{2} \mathrm{O}$ and $\mathrm{F}$ within the magma chamber, as discussed in section 5.1.

The following starting parameters were used for the best-fit model-calculation presented in Fig. 10: $\mathrm{H}_{2} \mathrm{O} 2.1 \mathrm{wt} \%$, F $0.3 \mathrm{wt} \%, \mathrm{Cl} 800 \mathrm{ppm}, \mathrm{W} 20 \mathrm{ppm}$ and Sn $20 \mathrm{ppm}$. Of these values, only Sn and $\mathrm{W}$ could be determined by LA-ICP-MS analysis of early (i.e., relatively unfractionated) melt inclusions in the Mole Granite (Table 1). The concentrations of $\mathrm{F}$ and $\mathrm{Cl}$ determined by EPMA in the melt inclusions in topaz ( $\mathrm{F} 2.5 \mathrm{wt} \%, \mathrm{Cl} 2500 \mathrm{ppm}$ ) are likely to be much higher than in the starting melt, as these inclusion were trapped at a very high degree of crystallization with respect to the whole magma chamber. The chosen values of $\mathrm{F}, \mathrm{H}_{2} \mathrm{O}$ and $\mathrm{Cl}$ thus represent a "best fit" between melt inclusion data published in Webster et al. (1996), and optimal reproducibility of the observed phenomena. Webster et al. (1996) report the following values for early melt inclusions from the basal vitrophyre of the Cerro el Lobo complex in Mexico: $\mathrm{H}_{2} \mathrm{O} 2.4 \pm 0.5 \mathrm{wt} \%$, F $0.35 \pm 0.4$ wt $\%, \mathrm{Cl} 1400 \pm 200 \mathrm{ppm}$.

\subsection{The chemical and hydrodynamic evolution of a crystallizing F-rich granitic sill}

Worster et al. (1990) have modeled the physical processes during the crystallization of a silllike, convecting magma chamber. Their results indicate that crystallization takes place to a larger degree in the center and at the bottom of the chamber rather than near the roof, even if the magma is cooled solely from above. This effect is most prominent in large sills because a comparatively larger fraction of heat can be transported by internal convection. Worster et al. (1990) assumed that only one layer of convection cells develops in a sill-like magma chamber, which is consistent with the conclusions of Spera et al. (1986). The crystallization process on a smaller scale is described by Huppert et al. (1986). Tank experiments demonstrate that the crystallization in a cooling magma chamber does not strictly proceed from the wall inwards, but starts in a mushy zone at some distance away from it. The less dense, residual melt rises up along the walls, forming a layer of more fractionated magma in the apical portions of the intrusion. Mahood and Cornejo (1992) demonstrated convincingly that this mechanism takes place also in nature.

Together with information taken from experimental phase diagrams presented in Whitney (1988), and phase relations reported from rhyolites (Christiansen et al., 1986; Webster and Duffield, 1994), the following evolution of the Mole Granite is proposed (Fig. 11): 

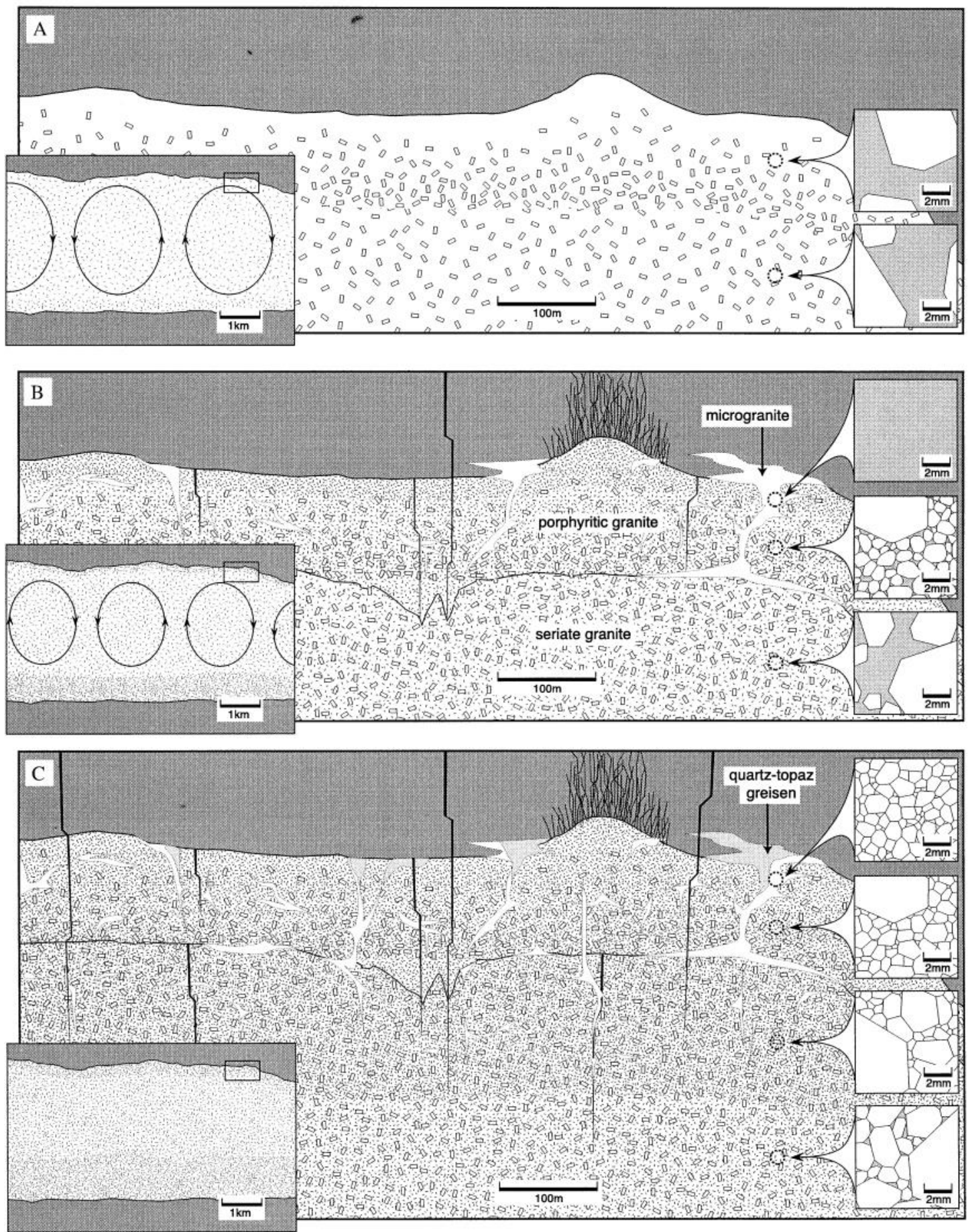

Figure 11. Cartoon section through the upper part of the Mole Granite at three stages of its crystallization history. (a) After the development of convection cells highly fractionated melt starts to accumulate under the roof of the magma chamber. The lower viscosity of this melt allows phenocrysts and mafic enclaves to settle down into the underlying, less fractionated melt portions. (b) Fluid saturation is reached at $\sim 40 \%$ crystallinity, leading to the formation of sheeted vein systems and a smaller grain size in the matrix of the porphyritic granite. The continuous exsolution of fluid results in the generation of cracks in the partly solidified carapace, and in the expulsion of interstitial melt into these zones of structural weakness. These melt portions will later crystallize to dikes and irregular masses of microgranite. (c) After $\sim 65 \%$ crystallinity is reached, the convection of the magma stops and the melt starts to crystallize by conduction. Fluids exsolving from F-rich residual melts in the contact zone form W-rich mineralizations and quartz-topaz greisens at the granite margin, whereas the Cl-rich fluids originating at greater depth form Sn-dominated deposits deeper within the granite. 
The magma of the Mole Granite has intruded at a depth of about $4 \mathrm{~km}(\approx 1 \mathrm{kbar})$, where it formed a large, sill-like magma body of 2-3 km thickness and $1800 \mathrm{~km}^{2}$ surface area1. At this time the melt had a temperature of $\sim 740{ }^{\circ} \mathrm{C}$, contained 25 vol\% phenocrysts (qtz, kfsp, plag and bio), and was with 3.5 wt $\% \mathrm{H}_{2} \mathrm{O}$ still fluid-undersaturated.

The development of convection cells shortly after magma emplacement resulted in a relatively homogeneous heat distribution within the magma chamber (Worster et al., 1990). Crystallization took place mainly in the interior and at the bottom of the chamber (see Huppert et al., 1986). Driven by the buoyancy force and large-scale convection cells the lighter, residual melt rose into apical regions of the magma chamber, where it formed a layer of more fractionated melt enriched in volatiles, F, Li, Rb, W, Sn, U and REE. The lower viscosity of this melt (Dingwell et al., 1993) caused already formed phenocrysts and mafic enclaves to settle down into the underlying, less fractionated portions of the magma (Fig. 11a).

Cooling down to $710^{\circ} \mathrm{C}$ was accompanied by approximately $15 \mathrm{vol} \%$ further crystallization, and the attainment of fluid saturation in the uppermost parts of the magma chamber. The latter resulted in an increase in the magma volume, leading to the formation of sheeted veins above ridges and bulges in the roof of the Mole Granite (Burnham, 1979). At fluid-saturated conditions $10{ }^{\circ} \mathrm{C}$ further cooling (or a 100 bar pressure-decrease) was enough to increase the degree of crystallization by additional 25 vol\% (Whitney, 1988). The associated increase in the crystallization rate lead to a finer grain-size in the groundmass of the porphyritic granite. The magma now had a total crystal content of about $65 \mathrm{vol} \%$, close to the value at which magma convection stops (Worster et al., 1990). Repeated build-up of overpressure in the magma chamber resulted in the expulsion of residual melts ( \pm fluid) into zones of structural weakness, where they crystallized to dikes and irregular masses of microgranite (Fig. 11b).

The one-phase fluids exsolving from fluorine-rich residual melts in the upper part of the magma chamber contained high amounts of tungsten and fluorine, and were responsible for the formation of $\mathrm{F}$ - and $\mathrm{W}$-rich ( $\pm \mathrm{Bi}, \mathrm{As}, \mathrm{Mo}, \mathrm{Be})$ ore veins along the granite margin. Quartz-topaz greisen preferentially developed within (or adjacent to) microgranites, as these were the last rocks to crystallize and therefore acted as channelways for the upward migrating fluids. At the stage shown in Fig. 11c interstitial melt is left only in the lower part of the seriate granite. Compared to the bulk mass of the 2-3 km thick intrusion these melts are still enriched in F, Li, Rb, W, Sn, U and REE, but not to the same degree as in the microgranites. The higher salinities of the fluids exsolving from these melts resulted in a strong fractionation of Cl-complexed elements $(\mathrm{Sn} \pm \mathrm{Pb}, \mathrm{Cu}, \mathrm{Zn}$ and $\mathrm{Ag}$ ) into the fluid, and correspondingly smaller enrichment factors (or even depletion) of these elements in the melt. The same element association is characteristic for deposits deeper within the Mole Granite.

\section{Conclusions}

Three groups of conclusions from this study relate to: (a) the technical aspect of investigating melt inclusions in plutonic systems, (b) the role of $\mathrm{F}$ in the magmatic-to-hydrothermal evolution of Sn-W mineralized granite systems, and (c) to the source processes of magmatic-hydrothermal systems in general.

Combined petrographic and microanalytical investigations on crystallized melt inclusions and coexisting fluid inclusions are a powerful tool to reconstruct the magmatic to hydrothermal transition of granitic plutons. LA-ICP-MS is particularly suited for the analysis of crystallized melt inclusions because it allows reconstitution of bulk element ratios by integration of transient

${ }^{1}$ all quantitative specifications in this section are associated with an uncertainty of about $20-30 \%$, except for the temperatures, which should be correct within 10\%) 
signals without physical re-homogenization prior to analysis. This simplifies preparation, and particularly reduces the risk of obtaining misleading results due to incomplete re-homogenization. Complete re-homogenization of melt inclusions is generally impossible if some volatile components were lost after trapping. This was found to be common in inclusions hosted by quartz, which had lost some $\mathrm{Na}$, but retained characteristic trace-element ratios. Melt inclusions in topaz survived without compositional modification in most samples, and could be re-homogenized at plausible temperatures.

The study of successive generations of fluid and melt inclusions from the Mole Granite indicates that the ore-metal concentrations of the magmatic-hydrothermal fluids were controlled by evolving concentrations of $\mathrm{H}_{2} \mathrm{O}, \mathrm{Cl}$ and $\mathrm{F}$ in the melt during a period of progressive fractional crystallization and concurrent fluid exsolution. Fluorine seems to have played the key role in this process, determining the salinity of the exsolving fluids through the F-dependent fluid - melt distribution coefficient of $\mathrm{Cl}$. Fluorine also facilitated the strong enrichment of $\mathrm{W}, \mathrm{Sn}, \mathrm{Th}, \mathrm{U}$ and other incompatible high-field-strength elements in the residual melt, as a result of lowered crystal - melt partition coefficients, and by protracting the crystal fractionation due to lowered solidus temperature and melt viscosity. Chlorine concentration in the fluid controlled the fluid - melt partition coefficients of Cl-complexed ore-forming metals such as Sn. Early Cl-rich fluids (occurring as brine coexisting with a separate vapor phase) were relatively Sn-rich. Further crystallization enriched the residual melt in $\mathrm{F}$, which reduced the tendency for $\mathrm{Cl}$ to partition into the fluid. The latest exsolving fluids were thus of moderately low salinity and single phase, and able to transport a larger concentration of $\mathrm{W}$ compared to Sn. Fluids with contrasting Sn/W ratio may have emanated concurrently from different levels within the fractionating granite pluton, enabling simultaneous formation of cassiterite-dominated and wolframite dominated vein deposits in the solidified carapace.

More generally, this study indicates that the chemistry of residual melts, rather than bulk magma compositions (represented by the final rock), control the composition of evolving magmatic-hydrothermal fluids. The effect of fluorine upon the fluid - melt partition coefficients of $\mathrm{Cl}$ and $\mathrm{Cl}$-complexed metals illustrates the complexity of the magmatic source control on metal ratios of the fluids. The study also emphasizes the importance of experimental data, which are indispensable to understand these relationships. Similar complexities due to apparently secondorder effects of slightly variable volatilities of $\mathrm{H}_{2} \mathrm{O}, \mathrm{Cl}, \mathrm{SO}_{2}$ and $\mathrm{H}_{2} \mathrm{~S}$ are likely to affect the $\mathrm{Fe}-\mathrm{Cu}-$ $\mathrm{Au}-\mathrm{Mo}-\mathrm{S}$ - redox relations in the calcalkaline source of porphyry-style ore deposits.

Acknowledgments. We would like to thank Eric Reusser for his help with the electron microprobe analyses, Rolf Frischknecht and Urs Menet for their assistance in developing the prototype LA-ICP-MS system at ETH Zürich, and Alex Riches and Sven Girsperger for handling the cold-seal bombs. The manuscript benefited from critical discussions with Thomas Pettke, Thomas Ulrich and Bob Bodnar, as well as from reviews by Ian Plimer, Doone Wyborn and an anonymous reviewer. Finally, we would like to thank Paul Ashley, Harvey Henley and Jim Stroud for their kind support during the field work in Australia. This research was funded by ETH Research Grant 0-20041-95 and by the Swiss National Science Foundation.

\section{References}

Anderson A. T. J. (1991) Hourglass inclusions: Theory and application to the Bishop rhyolitic Tuff. Am Mineral. 76, 530-547.

Audétat A., Günther D., and Heinrich C. A. (1998) Formation of a magmatic-hydrothermal ore deposit: Insights with LA-ICP-MS analysis of fluid inclusions. Science 279, 2091-2094. 
Audétat A. and Günther D. (1999) Mobility and $\mathrm{H}_{2} \mathrm{O}$-loss from fluid inclusions in natural quartz crystals. Contrib. Mineral. Petrol., in press.

Audétat A. (1999) The magmatic-hydrothermal evolution of the Sn/W-mineralized Mole Granite (Eastern Australia). unpubl. Ph.D. dissertation, ETH Zürich, Switzerland.

Audétat A., Günther D., and Heinrich C. A. (1999) Causes for large-scale metal zonation around mineralized plutons: Fluid inclusion LA-ICP-MS evidence from the Mole Granite, Australia. Econ. Geol., submitted.

Barnes H. L. (1997) Geochemistry of hydrothermal ore deposits. Wiley.

Barnes R. G., Brown R. E., Brownlow J. W., Gilligan L. B., Krynen J., and Willis I. L. (1988) A review of the mineral deposits of the New England Orogen in New South Wales. In New England Orogen; Tectonics and Metallogenesis (ed. J. D. Kleeman), pp. 211-227. University of New England.

Blevin P. L. and Chappell B. W. (1992) The role of magma sources, oxidation states and fractionation in determining the granite metallogeny of eastern Australia. Transact. Royal Soc. Edinburgh: Earth Sciences 83, 305-316.

Bodnar R. J. and Vityk M. O. (1994) Interpretation of microthermometric data for $\mathrm{H}_{2} \mathrm{O}-\mathrm{NaCl}$ fluid inclusions. In Fluid inclusions in minerals: methods and applications (ed. B. D. Vivo and M. L. Frezzotti), pp. 117-130. Virginia Tech.

Brodie R. (1983) Geology and mineralisation of the Mole River-Silent Grove area, near Tenterfield, Northern New South Wales. unpubl. BSc thesis, University of New England.

Burnham C. W. (1979) Magmas and hydrothermal fluids. In Geochemistry of hydrothermal ore deposits (ed. H. L. Barnes), pp. 71-136. Wiley.

Burnham C. W. and Ohmoto H. (1980) Late-stage processes of felsic magmatism. In Granitic magmatism and related mineralization, Vol. 8 (ed. S. Ishihara and S. Takenouchi), pp. 1-13. Mining Geol. Spec. Issue.

Candela P. A. and Holland H. D. (1986) A mass transfer model for copper and molybdenum in magmatic-hydrothermal systems; the origin of porphyry-type ore deposits. Econ. Geol. 81, 1-9.

Candela P. A. (1992) Controls on ore metal ratios in granite-related ore systems: an experimental and computational approach. Transactions of the Royal Society of Edinburgh: Earth Sciences 83, 317-326.

Candela P. A. and Piccoli P. M. (1995) Model ore-metal partitioning from melts into vapor and vapor/brine mixtures. In Magmas, Fluids and Ore Deposits, Vol. 23 (ed. J. F. H. Thompson), pp. 101127. Mineralogical Association of Canada, Short Course Series.

Carroll M. R. and Webster J. D. (1994) Solubilities of Sulfur, Noble Gases, Nitrogen, Chlorine, and Fluorine in Magmas. In Volatiles in Magmas, Vol. 30 (ed. M. R. Carroll and J. R. Holloway), pp. 251279. Mineralogical Society of America.

Christiansen E. H., Sheridan M. F., and Burt D. M. (1986) The geology and geochemistry of Cenozoic topaz rhyolites from the Western United States. Geological Society of America, Special Paper 205.

Cline J. S. and Bodnar R. J. (1991) Can economic porphyry copper mineralization be generated by a typical calc-alkaline melt? Journal of Geophysical Research 96, 8113-8126.

Cozens G. J. (1984) The geology and mineralisation of the southwest Mole Granite region, Northeastern New South Wales. unpubl. BSc Honours thesis, University of New England.

Creech M. (1992) Geology and Geochemistry of the Silexite Deposits of the Mole Granite, New England, NSW, and their potential economic significance. unpubl. MSc thesis, University of Technology, Sydney.

Didier J. and Babarin B. (1991) Enclaves and Granite Petrology. Elsevier, Amsterdam.

Dingwell D. B., Scarfe C. M., and Cronin D. J. (1993) The effect of F on viscosities in the system $\mathrm{Na}_{2} \mathrm{O}-\mathrm{Al}_{2} \mathrm{O}_{3}-\mathrm{SiO}_{2}$ : Implications for phonolites, trachytes and rhyolites. Am. Mineral. 70, 80-87. 
Dostal J. and Chatterjee A. K. (1995) Origin of topaz-bearing and related peraluminous granites of the Late Devonian Davis Lake pluton, Nova Scotia, Canada: crystal versus fluid fractionation. Chemical Geology 123, 67-88.

Dunbar N. W. and Hervig R. L.(1992) Petrogenesis and volatile stratigraphy of the Bishop Tuff: Evidence from melt inclusion analysis. J. Geophys. Res. 97, 15129-15150.

Eadington P. J. (1977) A study of fluid inclusions and their significance in minerals from hydrothermal ore deposits, New England, New South Wales. unpubl. Ph.D. thesis, University of Newcastle.

Eadington P. J. (1983) A fluid inclusion investigation of ore formation in a tin-mineralized granite, New England, New South Wales. Economic Geology 78, 1204-1221.

Eadington P. J. and Nashar B. (1978) Evidence for the Magmatic Origin of Quartz-Topaz Rocks From the New England Batholith, Australia. Contrib. Mineral. Petrol. 67, 433-438.

Fergusson C. L. and Leitch E. C. (1993) Late Carboniferous to Early Triassic Tectonics of the New England Fold Belt, Eastern Australia. In New England Orogen NEO'93 Conference Proceedings (eds. Flood P. G and Aitchison J. C.), pp. 53-59.

Flinter B. H., Hesp W. R., and Rigby D. (1972) Selected geochemical, mineralogical and petrological features of granitoids of the New England complex, Australia, and their relation to Sn, W, Mo and Cu mineralization. Economic Geology 67, 1241-1262.

Flood P. G. and Aitchison J. C. (1993) Recent advances in understanding the geological development of the New England Province of the New England Orogen. In New England Orogen NEO'93 Conference Proceedings (eds. Flood P. G and Aitchison J. C.), pp. 61-67.

Frezzotti M. L. (1992) Magmatic immiscibility and fluid phase evolution in the Mount Genis granite (SE Sardinia, Italy). Geochim. Cosmochim. Acta 56, 21-33.

Gebauer D. (1996) A P-T-t-path for an (ultra?-)high-pressure ultramafic/mafic rock association and its felsic country-rocks based on SHRIMP-dating of magmatic and metamorphic zircon domains. Example: Alpe Arami (Central Swiss Alps). In Earth processes: reading the Isotopic code (ed. unknown), pp. 309-328. American Geophysical Union.

Godden N. L. (1982) The volcanic-plutonic association, Tenterfield region, New South Wales. In New England Geology (ed. P. G. Flood and B. Runnegar), pp. 71-78. University of New England.

Günther D., Audétat A., Frischknecht R., and Heinrich C. A. (1998) Quantitative analysis of major, minor and trace elements in fluid inclusions using Laser Ablation-Inductively Coupled PlasmaMass Spectrometry (LA-ICP-MS). J. Anal. Atom. Spectrom. 13, 263-270.

Günther D., Frischknecht R., Heinrich C. A., and Kahlert H. J. (1997) Capabilities of an Argon Fluoride 193nm Excimer Laser for Laser Ablation Inductively Coupled Plasma Mass Spectrometry Microanalysis of Geological Materials. J. Anal. Atom. Spectrom. 12, 939-944.

Hedenquist J. W. and Lowenstern J. B. (1994) The role of magmas in the formation of hydrothermal ore deposits. Nature 370, 519-527.

Heinrich C. A. (1990) The chemistry of hydrothermal tin-tungsten ore deposition. Economic Geology 90, 705-729.

Heinrich C. A. (1995) Geochemical evolution and hydrothermal mineral deposition in Sn (-Wbasemetal) and other granite-related ore systems: some conclusions from Australian examples. In Magmas, Fluids and Ore Deposition, Vol. 23 (ed. J. F. H. Thompson). Mineralogical Association of Canada, Short Course Series.

Heinrich C. A., Günther D., Audétat A., Ulrich T., and Frischknecht R. (1999) Metal fractionation between magmatic brine and vapor, determined by micro-analysis of fluid inclusions. Geology 27, 755-758.

Heinrich C. A., Ryan C. G., and P. M. Ternagh (1992) Segregation of ore metals between magmatic brine and vapor: a fluid inclusion study using PIXE microanalysis. Econ. Geol. 87, 1566-1583.

Heinrich D. A. and Ryan C. G. (1992) Mineral paragenesis and regional zonation of graniterelated $\mathrm{Sn}-\mathrm{As}-\mathrm{Cu}-\mathrm{Pb}-\mathrm{Zn}$ deposits: A chemical model for the Mole Granite district (Australia) based 
on PIXE fluid inclusion analyses. In Water-Rock Interaction. Proceedings of the 7th intern. symp. on water-rock interaction, Park City, USA, 13-18 July 1992. (ed. Y. K. Kharaka and A. S. Maest), pp. 15831587. Balkema.

Henley H. F., Brown R. E., and Stroud W. J. (1999) The Mole Granite - extent of mineralisation and exploration potential. In Regional geology, tectonics and metallogenesis of the New England Orogen (ed. P. G. Flood), pp. 385-392. University of New England, Armidale, Australia.

Hensel H. D., McCulloch M. T., and Chappell B. W. (1985) The New England Batholith: constraints on its derivation from $\mathrm{Nd}$ and $\mathrm{Sr}$ isotopic studies of granitoids and country rocks. Geochim. Cosmochim. Acta 42, 369-384.

Hildreth W. (1981) Gradients in silicic magma chambers: Implications for lithospheric magmatism. J. Geophys. Res. 86, 10153-10192.

Huppert H. E. and Sparks R. S. J. (1988) The Generation of Granitic Magmas by Intrusion of Basalt into Continental Crust. Journal of Petrology 29(3), 599-624.

Huppert H. E., Sparks R. S. J., Wilson J. R., and Hallworth M. A. (1986) Cooling and crystallization at an inclined plane. Earth and Planetary Science Letters 79, 319-328.

Ishihara S. and Takenouchi S. (1980) Granitic magmatism and related mineralization. Mining Geology Special Issue, Vol. 8. The Society of Mining Geologists of Japan.

Johannes W. and Holtz F. (1996) Petrogenesis and experimental petrology of granitic rocks. Springer.

Juniper D. N. and Kleeman J. D. (1979) Geochemical characterization of some tin-mineralizing granites of New South Wales. J. Geochemical Exploration 11, 321-333.

Keppler H. (1993) Influence of fluorine on the enrichment of high field strength trace elements in granitic rocks. Contribution to Mineralogy and Petrology 114, 479-488.

Keppler H. and Wyllie P. J. (1991) Partitioning of $\mathrm{Cu}, \mathrm{Sn}, \mathrm{Mo}, \mathrm{W}, \mathrm{U}$ and Th between melt and aqueous fluid in the systems haplogranite- $\mathrm{H}_{2} \mathrm{O}-\mathrm{HCl}$ and haplogranite- $\mathrm{H}_{2} \mathrm{O}-\mathrm{HF}$. Contrib. Mineral. Petrol. 109, 139-150.

Kesler S. E. (1994) Mineral resources, economics and the environment. McMillan.

Kleeman J. D. (1982) The anatomy of a tin-mineralizing A-type granite. In New England Geology (ed. P. G. Flood and B. Runnegar), pp. 327-334. University of New England.

Kleeman J. D. (1985) Origin of disseminated wolframite-bearing quartz-topaz rock at Torrington, New South Wales, Australia. In High heat flow production (HHP) granites, hydrothermal circulation and ore genesis, pp. 197-201. Institution of Mining and Metallurgy.

Kleeman J. D., Plimer I. R., Lu J., Foster D. A., and Davidson R. (1997) Timing of thermal and mineralization events associated with the Mole Granite, New South Wales. In Tectonics and metallogenesis of the New England Orogen, Special Publication 19 (ed. P. M. Ashley and P. G. Flood), pp. 254-265. Geological Society of Australia.

Laemmlein G. (1930) Korrosion und Regeneration der Porphyr-Quarze. Zeitschrift für Kristallographie 75, 109-127.

Lange R. A. (1994) The effect of $\mathrm{H}_{2} \mathrm{O}, \mathrm{CO}_{2}$ and $\mathrm{F}$ on the density and viscosity of silicate melts. In Volatiles in Magmas, Vol. 30 (ed. M. R. Carroll and J. R. Holloway), pp. 331-369. Mineralogical Society of America.

Lehmann B. (1990) Metallogeny of Tin. Springer.

London D. (1987) Internal differentiation of rare-element pegmatites; Effects of boron, phosphorus and fluorine. Geochim. Cosmochim. Acta 51, 403-420.

London D. (1996) Granitic pegmatites. Trans. Royal Soc. Edinburgh: Earth Sciences 87, 305-319.

London D., Morgan G. B., and Hervig R. L. (1989) Vapor-undersaturated experiments with Macusani-glass $+\mathrm{H}_{2} \mathrm{O}$ at $200 \mathrm{MPa}$, and the internal differentiation of granititc pegmatites. Contrib. Mineral. Petrol. 102, 1-17.

Lonergan A. D. (1971) Ore deposits in the southern half of the Mole Tableland. unpubl. BSc Honours thesis, University of New England. 
Lowenstern J. B. (1994) Chlorine, fluid immiscibility, and degassing in peralkaline magmas from Pantelleria, Italy. Amer. Mineral. 79, 353-369.

Lowenstern J. B. (1995) Application of silicate-melt inclusions to the study of magmatic volatiles. In Magmas, fluids and ore deposition, Mineralogical Assoc. of Canada, Short Course Series Vol. 23 (ed. J. F. H. Thompson), pp. 71-98. Mackenzie D. E., Black L. P., and Sun S. S. (1988) Origin of alkali-feldspar granites: An example from the Poimena Granite, northeastern Tasmania, Australia. Geochim. Cosmochim. Acta 52, 2507-2524.

Mader H. M., Zhang Y., Phillips J. C., Sparks R. S. J., and Sturtevant B.(1994) Experimental simulation of explosive degassing of magma. Nature 372, 85-88.

Mahood G. A. and Cornejo P. C. (1992) Evidence for ascent of differentiated liquids in a silicic magma chamber found in a granitic pluton. Transact. Royal Soc. Edinburgh: Earth Sciences 83, 6369.

Manning D. A. C. and Henderson P. (1984) The behavior of tungsten in granitic melt-vapor systems. Contrib. Mineral. Petrol. 86, 286-293.

Marshall D. J. (1988) Cathodoluminescence of geological materials. Unwin Hyman.

McClatchie L. (1981) Preliminary report on mapping programme, Torrington roof pendant, Northern New South Wales, pp. 1-8. GS Report, Geological Survey of New South Wales.

McPhie J. (1988) Source(s) of the Dundee Rhyodacite ignimbrite: implications for the existence of Late Perminan cauldrons in the southern New England Orogen. In New England Orogen; Tectonics and Metallogenesis (ed. J. D. Kleeman), pp. 145-149. University of New England.

Mulholland C. S. J. (1943) Torrington Tin and Wolfram Deposits, pp. 1-23. GS Report, Geological Survey of New South Wales.

Nekvasil H. (1991) Ascent of felsic magmas and formation of rapakivi. Am. Mineral. 76, 12791290.

Plimer I. R. and Kleeman J. D. (1985) Mineralization associated with the Mole Granite, Australia. In High heat production granites (HHP), hydrothermal circulation and ore genesis, pp. 563-569. Institution of Mining and Metallurgy.

Rankin A. H., Ramsey M. H., Coles B., Langevelde F. V., and Thomas C. R. (1992) The composition of hypersaline, iron-rich granitic fluids based on laser-ICP and Synchrotron-XRF microprobe analysis of individual fluid inclusions in topaz, Mole Granite, Eastern Australia. Geochim. Cosmochim. Acta 56, 67-79.

Rapien M. H., Bodnar R. J., Czabo C., Simmons S., and Wood C. P. (1997) Magma evolution of White Island, New Zealand; evidence from melt inclusions. AGU 1997 spring meeting, 330-331.

Sardjono A. (1988) Airborne radiometric mapping of the Mole Tableland, northern New South Wales. unpubl. Masters thesis, University of New England.

Sawkins F. J. (1990) Metal deposits in relation to plate tectonics. Springer.

Shaw S. E. and Flood R. H. (1981) The New England Batholith, Eastern Australia: Geochemical variations in time and space. J. Geophys. Research 86, 10530-10544.

Shaw S. E. and Flood R. H. (1993) A compilation of Late Permian and Triassic biotite Rb-Sr Data from the New England Batholith and areas to the Southeast. In CIS Research Report 1991-1992 (ed. P. F. Carr), pp. 151-155. CSIRO Mineral Research Laboratories.

Shaw S. E., Flood R. H., and Vernon R. H. (1988) Structure of the Dundee Ignimbrite. In New England Orogen; Tectonics and Metallogenesis (ed. J. D. Kleeman), pp. 150-156. University of New England.

Shinohara H. (1994) Exsolution of immiscible vapor and liquid phases from a crystallizing silicate melt: Implications for chlorine and metal transport. Geochim. Cosmochim. Acta 58(23), 5215-5221.

Shinohara H. and Hedenquist J. W. (1997) Constraints on magma degassing beneath the Far Southwest porphyry $\mathrm{Cu}$-Au deposit, Philippines. J. Petrol. 38, 1741-1752 
Skirius C. M., Peterson J. W., and Anderson A. T. Jr. (1990) Homogenizing rhyolitic glass inclusions from the Bishop Tuff. Am. Mineral. 75, 1381-1398.

Spera F. J., Yuen D. A., Clark S., and Hong H. J. (1986) Double-diffusive convection in magma chambers: single or multiple layers? Geophys. Research Letters 13(1), 153-156.

Stegman C. L. (1983) The Mole Granite and its Sn-W-Mo-basemetal mineralization - a study of its southern-central margin. unpubl. BSc Honours thesis, University of New England.

Stein H. J. and Hannah J. L. (1990) Ore-bearing Granite Systems. Geological Society of America, Special Papers.

Student J. J. and Bodnar R. J. (1996) Melt inclusion microthermometry: Petrologic constraints from the H2O-saturated haplogranite system. Petrology 4, 291-306.

Sun S. S. and Eadington P. J. (1987) Oxygen isotope evidence for the mixing of magmatic and meteoric waters during tin mineralization in the Mole Granite, New South Wales, Australia. Economic Geology 85, 43-52.

Taylor J. R. and Wall V. J. (1984) The mobilisation of tin from granitoid magmas. 27th Int. Geol. Congr. Moscow, 474 (abstract).

Taylor R. P., Jackson S. E., Longerich H. P., and Webster J. D. (1997) In situ trace-element analysis of individual silicate melt inclusions by laser ablation microprobe -inductively coupled plasmamass spectrometry (LAM-ICP-MS). Geochim. Cosmochim. Acta 61, 2559-2567.

Thompson J. F. H. (1995) Magmas, Fluids and Ore Deposition. Mineralogical Association of Canada, Short Course Series.

Thomas R., Rhede D., and Trumbull R. B., (1996) Microthermometry of volatile-rich silicate melt inclusions in granitic rocks. Z. geol. Wiss. 24, 505-526.

Ulrich T., Günther D., and Heinrich C. A. (1999) Gold concentrations of magmatic brines and the metal budget of porphyry copper deposits. Nature 399, 676-679.

Urabe T. (1985) Aluminous granite as a source of hydrothermal ore deposits: an experimental study. Economic Geology 80, 148-157.

Vernon R. H. (1986) K-feldspar megacrysts in granites - phenocrysts, not porphyroblasts. Earth-Sciences Reviews 23, 1-63.

Vickery N. M., Ashley P. M., and Fanning C. M. (1997) Dumboy-Gragin Granite, northeastern New South Wales: age and compositional affinities. In Tectonics and metallogenesis of the New England Orogen (ed. Flood P. F.), pp. 266-271. Geol. Soc. Australia Special Publ. 19.

Vivo B. D. and Frezzotti M. L. (1994) Evidence for magmatic immiscibility in Italian subvolcanic systems. In Fluid inclusions in Minerals: Methods and Applications (ed. B. D. Vivo and M. L. Frezzotti), pp. 345-362. Virginia Tech.

Wallace P. J., Anderson A. T. Jr., and Davis A. M. (1999) Gradients in $\mathrm{H}_{2} \mathrm{O}, \mathrm{CO}_{2}$, and exsolved gas in a large-volume silicic magma system: Interpreting the record preserved in melt inclusions from the Bishop Tuff. J. Geophys. Res. 104, 20097-20122.

Weber C. R. (1974) Woolomin-Texas Block: Plutonic rocks and intruded sediments. In The Mineral Deposits of New South Wales (ed. N. L. Markham and H. Basden), pp. 352-391. Geological Survey of New South Wales.

Webster J. D., Burt D. M., and Aguillon R. A. (1996) Volatile and lithophile trace-element geochemistry of Mexican tin rhyolite magmas deduced from melt inclusions. Geochim. Cosmochim. Acta 60(17), 3267-3284.

Webster J. D. and Duffield W. A. (1994) Extreme halogen abundances in tin-rich magma of the Taylor Creek Rhyolite, New Mexico. Economic Geology 89, 840-850.

Webster J. D. and Holloway J. R. (1990) Partitioning of $\mathrm{F}$ and $\mathrm{Cl}$ between magmatic hydrothermal fluids and highly evolved granitic magmas. In Ore-bearing granite systems; Petrogenesis and mineralizing processes, Vol. 246 (ed. H. J. Stein and J. L. Hannah), pp. 21-34. Geological Society of America, Special Paper. 
Webster J. D., Thomas R., Rhede D., Förster H. J., and Seltmann R. (1997) Melt inclusions in quartz from an evolved peraluminous pegmatite: Geochemical evidence for strong tin enrichment in fluorine-rich and phosphorus-rich residual liquids. Geochim. Cosmochim. Acta 61, 2589-2604.

Whitney J. A. (1988) The origin of granites: The role and source of water in the evolution of granitic magmas. Geol. Soc. Am. Bull. 100, 1886-1997.

Wood S. A. and Vlassopoulos D. (1989) Experimental determination of the hydrothermal solubility and speciation of tungsten at $500^{\circ} \mathrm{C}$ and $1 \mathrm{kbar}$. Geochim. Cosmochim. Acta 53, 303-312.

Worster M. G., Huppert H. E., and Sparks R. S. J. (1990) Convection and crystallization in magma cooled from above. Earth and Planetary Science Letters 101, 78-89.

Yang K. and Bodnar R. J. (1994) Magmatic-hydrothermal evolution in the "bottoms" of porphyry copper systems; evidence from silicate melt and aqueous fluid inclusions in granitoid intrusions in the Gyesongsang Basin, South Korea. International Geology Review 36(7), 608-628.

Zilong C. and Shenglin P. (1994) The experimental results of W and Sn partitioning between fluid and melt and their significance for the origin of W and Sn ore deposits (in Chinese). Dizhi Lunping 40(3), 274-282. 